\title{
Numerical investigation of particle velocity distributions in aeolian sand transport
}

\author{
Liqiang Kang a,b,*, Dayou Liu ${ }^{c}$ \\ a State Key Laboratory of Earth Surface Processes and Resource Ecology, Beijing Normal University, Beijing 100875, China \\ ${ }^{\mathrm{b}}$ MOE Engineering Research Center of Desertification and Blown-sand Control, Beijing Normal University, Beijing 100875, China \\ c Center for Plasma and Combustion Research, Institute of Mechanics, Chinese Academy of Sciences, Beijing 100190, China
}

\section{A R T I C L E I N F O}

Article history:

Received 7 May 2009

Received in revised form 29 September 2009

Accepted 4 October 2009

Available online 14 October 2009

\section{Keywords:}

Aeolian sand transport

Particle velocity

Probability distribution

Simulation

\begin{abstract}
A B S T R A C T
Particle velocity distribution in a blowing sand cloud is a reflection of saltation movement of many particles. Numerical analysis is performed for particle velocity distribution with a discrete particle model. The probability distributions of resultant particle velocity in the impact-entrainment process, particle horizontal and vertical velocities at different heights and the vertical velocity of ascending particles are analyzed. The probability distributions of resultant impact and lift-off velocities of saltating particles can be expressed by a log-normal function, and that of impact angle comply with an exponential function. The probability distribution of particle horizontal and vertical velocities at different heights shows a typical single-peak pattern. In the lower part of saltation layer, the particle horizontal velocity distribution is positively skewed. Further analysis shows that the probability density function of the vertical velocity of ascending particles is similar to the right-hand part of a normal distribution function, and a general equation is acquired for the probability density function of non-dimensional vertical velocity of ascending particles which is independent of diameter of saltating particles, wind strength and height. These distributions in the present numerical analysis are consistent with reported experimental results. The present investigation is important for understanding the saltation state in wind-blown sand movement.
\end{abstract}

(c) 2009 Elsevier B.V. All rights reserved.

\section{Introduction}

Sand transport by wind is a type of movement of many sand grains. The movement of particles in blowing sand transport is classified to three processes (creep, saltation and suspension), of which saltation, in which sand grains are propelled by wind along the surface in short hops, is the dominant mode of blown sand movement, accounting for about $75 \%$ of the total sand flux (Bagnold, 1941). Sand movement in saltation cloud is very complex and related to many impact factors such as wind velocity and inter-particle collisions in air. Hence, the particle velocity in a blowing sand cloud at a fixed height is not a constant and can show a particular probability distribution pattern. The particle velocity distributions in a blowing sand cloud are important to understand this saltation movement state.

In the last two decades some numerical models of aeolian sand transport have been developed and generally subdivided into several different sub-processes, such as aerodynamic entrainment, grain trajectories, grain-bed impacts, and wind field modification (Ungar and Haff, 1987; Anderson and Haff, 1988; Anderson and Haff, 1991; McEwan and Willetts, 1991, 1993; Spies et al., 2000; Spies and

\footnotetext{
* Corresponding author. State Key Laboratory of Earth Surface Processes and Resource Ecology, Beijing Normal University, Beijing 100875, China. Tel./fax: +86 10 62207162.

E-mail addresses: klq@sohu.com, kangliqiang@bnu.edu.cn (L. Kang).
}

McEwan, 2000). By linking these sub-processes, a negative feedback mechanism is established to form a saltating equilibrium state. In these numerical models, the grain-bed collision is generally treated by an empirical way; the splash function (Ungar and Haff, 1987; Anderson and Haff, 1988, 1991) or a set of the experimental data (McEwan and Willetts, 1991, 1993) is used to specify the initial or launch velocity distribution of saltating grains, which further affects the simulated saltation path heights and lengths. The initial lift-off velocity distribution of saltating grains is an important input parameter in these numerical models.

The lift-off velocity distribution of saltating grains is also a bridge to link the micro- and macro-scale research, and the particle velocity distribution in air is a reflection of saltation movement of many sand grains. Therefore, it is important to accurately describe the lift-off velocity distribution of saltating grains and the particle velocity distribution in air, which are helpful to estimate the statistical parameters of saltating grains.

By theoretical model, wind tunnel and field experiments, many efforts have been made to acquire the parameters about particle velocity distribution, such as the horizontal and vertical velocity distributions and the impact and lift-off velocity distributions (e.g. Willetts and Rice, 1986; Anderson and Haff, 1991; Nalpanis et al., 1993; Greeley et al., 1996; Zou et al., 2001; Dong et al., 2002; Namikas, 2003; Dong et al., 2004; Huang et al., 2006; Cheng et al., 2006).

Anderson and Haff $(1988,1991)$ used a Gaussian distribution for the rebound velocity of particles and an exponential function for the 
ejected velocity. Sørensen (1991) used a normal function to describe the distribution of the sand horizontal ejection velocity. Anderson and Hallet (1986) and Raupach (1991) described the initial vertical velocity by an exponential distribution. Namikas (2003) introduced the gamma and exponential launch velocity distributions into a numerical model.

In the experiments of Nalpanis et al. (1993), the probability distributions of ejection velocity and the ejection angle of sand grains are similar to the log-normal distribution. Greeley et al. (1996) measured the sand speed in a field by analysis of high-speed motion pictures of saltating grains; the results show that the velocity distributions of ascending and descending grains have a single peak, but the distribution pattern is not given. The wind tunnel experiment of Zou et al. (2001) gave the probability distribution of particle velocity at different heights as a Pearson VII distribution pattern.

Dong et al. $(2002,2004)$ first used the particle dynamic analyzer (PDA), which is a non-intrusive measurement using Doppler technique, to measure the blowing sand velocity in a wind tunnel. Dong et al. (2002) gave that the velocity distribution of impacting and entrained particles as described by a Weibull function, but the probability distribution of impacting and lift-off angles is complex and cannot be expressed by a simple function. Dong et al. (2004) found the probability distribution of the downwind sand velocity complies with a Gaussian function, while that of vertical velocity is a Lorentzian function for fine particles and complex for coarse particles.

Cheng et al. (2006) combined theoretical analyses with wind tunnel experiment data to describe the lift-off parameters of saltating grains. In their results the lift-off angles follow a LogNorm4 distribution function, whereas the horizontal, vertical, and resultant lift-off velocities follow a Gamma distribution function.

Zhang et al. (2007) used a high-speed digital camera to record the saltating trajectories in a wind tunnel. In their results, the probability distributions of resultant ejection velocity, ejection angle, horizontal and vertical ejection velocities show a single peak; the probability distributions of resultant impact velocity, impact angle and horizontal impact velocity also show a single peak, and the distribution of vertical impact velocity shows two sharp peaks for the smaller beach sand and the desert sand.

From the above investigations, it can be seen that the particle velocity distributions have many types of distribution patterns because of the complexity of the problem. It is necessary for us to correctly predict the particle velocity distributions on the bed surface and at different heights. Hence, further studies are needed.

Kang et al. (2008a,b,c) reported the particle velocity distributions from wind tunnel experiments. In this paper, further studies of particle velocity distributions are focused on the numerical analysis. The discrete particle simulation of aeolian sand transport was carried out by Kang and Guo (2006) to analyze the macro-scale parameters, such as the sand velocity profile, sand mass flux and wind velocity profile. In this paper, the discrete particle model of aeolian sand transport is applied to predict directly the particle velocity distributions (including resultant impact and lift-off velocities, particle horizontal and vertical velocities at different heights and the vertical velocity of ascending particles). Then, the mathematical model for sand transport by wind is described, the numerical method and simulated conditions are given, the simulated results and discussions are considered, and further analysis for vertical velocity distribution of ascending particles is discussed.

\section{Mathematical model}

The discrete particle model is applied to describe sand transport by wind. The model can give detailed information of particle motion in space at the individual particle level. Therefore, the particle velocity distributions can be directly deduced from the simulation results.

\subsection{Equations of gas phase}

Considering the Magnus force, the continuity and momentum equations of gas can be described as (based on the model of Kang and Guo, 2006):

$$
\begin{aligned}
& \frac{\partial}{\partial t}\left(\alpha_{f} \rho_{f}\right)+\nabla \cdot\left(\alpha_{f} \rho_{f} u_{f}\right)=0 \\
& \frac{\partial}{\partial t}\left(\alpha_{f} \rho_{f} u_{f}\right)+\nabla \cdot\left(\alpha_{f} \rho_{f} u_{f} u_{f}\right)= \\
& -\alpha_{f} \nabla p+\nabla \cdot\left(\alpha_{f} \tau_{f}\right)+\alpha_{f} \rho_{f} g-f_{\text {drag }}-f_{\text {Mag }}
\end{aligned}
$$

where $\rho_{f}, u_{f}$ and $p$ are the fluid density, velocity and pressure, respectively; $g$ is acceleration due to gravity; $\tau_{f}$ is the fluid shear stress; $\alpha_{f}$ is the volume fraction of fluid; $f_{\text {drag }}$ is the volumetric fluidparticle interaction force; and $f_{\text {Mag }}$ is the volumetric Magnus force.

$\tau_{f}, \alpha_{f}, f_{\text {drag }}$ and $f_{\text {Mag }}$ are expressed as follows:

$$
\begin{aligned}
& \tau_{f}=-\frac{2}{3}\left(\mu_{e f f} \nabla \cdot u_{f}\right) \delta_{k}+\mu_{e f f}\left[\nabla u_{f}+\left(\nabla u_{f}\right)^{T}\right] \\
& \alpha_{f}=1-\sum_{i=1}^{n} V_{p i} / \Delta V \\
& f_{\text {drag }}=\frac{1}{\Delta V} \sum_{i=1}^{n} F_{\text {drag }, i} \\
& f_{\text {Mag }}=\frac{1}{\Delta V} \sum_{i=1}^{n} F_{\text {Mag }, i}
\end{aligned}
$$

where $\mu_{\text {eff }}$ is the fluid effective viscosity; $\boldsymbol{\delta}_{k}$ is the Kronecker delta; $\Delta V$ and $V_{p i}$ are the volume of a computational cell and the volume of particle $i$ inside this cell, respectively; and $n$ is the number of particles in the cell. For the two-dimensional flow, $\Delta V=\Delta x \Delta y d_{p}, \Delta x$ and $\Delta y$ are the lengths of a computational cell in $x$ and $y$ directions, respectively, and $d_{p}$ is the particle diameter.

$F_{\text {drag }}$ is the fluid drag force on a particle, which can be described as (Di Felice, 1994):

$F_{\text {drag }}=\frac{C_{d 0}}{8} \pi d_{p}^{2} \rho_{f} \alpha_{f}^{2}\left|u_{f}-u_{p}\right|\left(u_{f}-u_{p}\right) \alpha_{f}^{-\chi}$

where $\chi=3.7-0.65 \exp \left[-\left(1.5-\log \mathrm{Re}_{p}\right)^{2} / 2\right] ; u_{p}$ is the translational velocity of particle; and $C_{d 0}$ and $\operatorname{Re}_{p}$ are the fluid drag coefficient and particle Reynolds number, respectively:

$C_{d 0}=\left(0.63+\frac{4.8}{R e_{p}^{0.5}}\right)^{2}$
$\operatorname{Re}_{p}=\frac{\alpha_{f} \rho_{f} d_{p}\left|u_{f}-u_{p}\right|}{\mu_{f}}$

$F_{M a g}$ is the Magnus force on a particle. The Magnus force is the lift force due to rotation of the particle. Rubinow and Keller (1961) derived the Magnus force for Reynolds numbers of the order of unity. Including the flow rotation effects, the Magnus force can be expressed as (Crowe et al., 1998):

$F_{\text {Mag }}=\frac{\pi}{8} d_{p}^{3} \rho_{f}\left[\left(\frac{1}{2} \nabla \times u_{f}-\omega_{p}\right) \times\left(u_{f}-u_{p}\right)\right]$

where $\frac{1}{2} \nabla \times u_{f}$ is the angular velocity of the local fluid rotation and $\omega_{p}$ is the angular velocity of particle.

In this paper, the fluid turbulence is treated with standard $k-\varepsilon$ turbulent model, $k$ is the turbulence kinetic energy and $\varepsilon$ is the 
dissipation rate of $k$. The transport equations of standard $k-\varepsilon$ turbulent model can be expressed as:

$\frac{\partial}{\partial t}\left(\alpha_{f} \rho_{f} k\right)+\nabla \cdot\left(\alpha_{f} \rho_{f} u_{f} k\right)=\nabla \cdot\left(\alpha_{f}\left(\mu_{f}+\frac{\mu_{\mathrm{ft}}}{\sigma_{k}}\right) \nabla k\right)+\alpha_{f} G-\alpha_{f} \rho_{f} \varepsilon$

$\frac{\partial}{\partial t}\left(\alpha_{f} \rho_{f} \varepsilon\right)+\nabla \cdot\left(\alpha_{f} \rho_{f} u_{f} \varepsilon\right)=\nabla \cdot\left(\alpha_{f}\left(\mu_{f}+\frac{\mu_{\mathrm{ft}}}{\sigma_{\varepsilon}}\right) \nabla \varepsilon\right)+\alpha_{f} \frac{\varepsilon}{k}\left(c_{1} G-c_{2} \rho_{f} \varepsilon\right)$

where $\mu_{f}$ and $\mu_{f t}$ are the fluid dynamic viscosity and turbulent viscosity, respectively. $\mu_{\text {eff }}=\mu_{f}+\mu_{f t}, \mu_{f t}=c_{\mu} \rho_{f} k^{2} / \varepsilon, G=\mu_{f t} \nabla u_{f} \cdot\left[\nabla u_{f}+\right.$ $\left.\left(\nabla u_{f}\right)^{T}\right] . c_{1}, c_{2}$ and $c_{\mu}$ are constants; $c_{1}=1.44, c_{2}=1.92$ and $c_{\mu}=0.09$. $\sigma_{k}$ and $\sigma_{\varepsilon}$ are the turbulent Prandtl numbers for $k$ and $\varepsilon$, respectively, $\sigma_{k}=1.0$ and $\sigma_{\varepsilon}=1.3$.

\subsection{Equations of particle motion}

The equations of particle translational and rotational motion are expressed by:

$m_{p} \frac{d u_{p}}{d t}=m_{p} g+F_{d r a g}+F_{M a g}+\sum_{j=1}^{n_{c}}\left(f_{n, i j}+f_{t, i j}\right)$

$I_{p} \frac{d \omega_{p}}{d t}=\sum_{j=1}^{n_{c}} T_{i j}+T_{f}$

where $m_{p}, u_{p}$ and $\omega_{p}$ are the mass, translational and angular velocity of particle $i$, respectively; $f_{n, i j}$ and $f_{t, i j}$ are the normal and tangential forces between particle $i$ and $j$ due to particle collision, respectively; $n_{c}$ is the number of the particles in contact with particle $i ; T_{i j}$ is the torque between particle $i$ and $j$ due to particle collision; and $I_{p}$ is the moment of inertia of particle, $I_{p}=\frac{1}{10} m_{p} d_{p}^{2}$.

$T_{f}$ is the flow torque acting on the particle in a fluid due to the shear stress distribution on the particle surface. For a low Reynolds number flow, the torque $T_{f}$ on a spherical particle can be expressed as (Happel and Brenner, 1973):

$T_{f}=\pi \mu_{f} d_{p}^{3}\left(\frac{1}{2} \nabla \times u_{f}-\omega_{p}\right)$

\subsection{Inter-particle collision model}

The soft sphere model is used to describe inter-particle collisions. The soft sphere model can treat the multiple particle contacts and give the information about the transfer of inter-particle forces ( $\mathrm{Xu}$ and $\mathrm{Yu}$, 1997; Mikami et al., 1998; Crowe et al., 1998).

The inter-particle forces can be described by a linear spring-damping model as follows (Crowe et al., 1998):

$f_{n, i j}=-k_{s} \delta_{n}-\eta v_{n, i j}$

$f_{t, i j}=\left\{\begin{array}{l}-k_{s} \delta_{t}-\eta v_{t, i j}, \quad\left|f_{t, i j}\right| \leq \mu_{s}\left|f_{n, i j}\right| \\ -\mu_{s}\left|f_{n, i j}\right| t, \quad\left|f_{t, i j}\right|>\mu_{s}\left|f_{n, i j}\right|\end{array}\right.$

where $k_{\mathrm{s}}$ and $\eta$ are the stiffness and damping coefficient, respectively; $\mu_{\mathrm{s}}$ is friction coefficient; $\delta$ is displacement vector between two contacting particles; $v$ is the relative velocity vector between two contacting particles, $v_{n, i j}=\left(v_{i j} \cdot \mathrm{n}\right) \mathrm{n}, v_{t, i j}=v_{i j}-v_{n, i j}, v_{i j}=v_{i}-v_{j}+\omega_{i} \times R_{i}-$ $\omega_{j} \times R_{j}, R$ is a vector from the mass center of particle to the contact point. $n$ is the unit vector from the center of particle $i$ to that of particle $j, n=R_{i}$ / $\left|R_{i}\right|$ and $t$ is the unit tangential vector, $t=v_{t, i j} /\left|v_{t, i j}\right|$.

\section{Numerical method and simulated conditions}

The equations of gas phase are solved by the conventional SIMPLEC (Semi-Implicit Method for Pressure-Linked Equations - Consistent) method. The finite volume method is applied to discretize the gas equations on a non-staggered rectangular grid. The second-order central difference scheme is used for the diffusion terms. The QUICK (Quadratic Upwind Interpolation of Convective Kinematics) scheme is used for the convective term of the momentum equation.

The motion equations of discrete particles are solved by the explicit time integration method.

In order to reduce the CPU time, for each particle the neighbor list is used to store all of neighbors and a check for possible collisions is performed only for the particles in this list. Fig. 1 shows the structure of the neighbor list. Using a grid method, each particle control volume (PCV) is defined. In Fig. 1, for the two-dimensional simulation, the neighbor list of the black particle includes all the other particles within the black particle PCV and its adjacent eight PCVs, hence, the collision search for this black particle is only limited to the black particle PCV and its eight neighbor PCVs. In order to avoid the repeated collision search, each particle is numbered, for the particle $i$ only the neighbor particle $j(j>i)$ ) in the neighbor list of particle $i$ is checked for collision search.

The three-dimensional simulation is better than the two-dimensional simulation, but the two-dimensional simulation can also reveal the characteristics of particle motion qualitatively. Moreover, the three-dimensional simulation needs more computational conditions. Hence, in this paper, the two-dimensional simulation is done for the particle velocity distribution.

The computational domain is a two-dimensional rectangular region. The structure of computational domain is shown in Fig. 2.

A periodic boundary condition is used for inlet and outlet. For particles, when particles leave the computational domain from the outlet, they will enter the computational domain via the inlet; when particles leave the computational domain from the inlet, they will enter the computational domain from the outlet. For air, at the end of each time step, the gas velocity $u_{\mathrm{f}}$, the turbulence kinetic energy $k$ and its dissipation rate $\varepsilon$ at the outlet are given to the corresponding grid cell of the inlet.

The inlet initial velocity of the air is a logarithmical function $u=\frac{u_{*}}{\kappa} \ln \left(\frac{y}{y_{0}}\right)$, where $\kappa$ is von Karman's constant, $\kappa=0.4, y_{0}$ is the roughness length, $u *$ is the friction velocity. In the initial state, the inlet turbulent intensity of the air is $3.7 \%$.

On the top boundary, the constant shear stress is set for the gas, the reflective boundary condition is used for particles. Hence, at the steady state, the wind velocity is mainly driven by the constant shear stress of

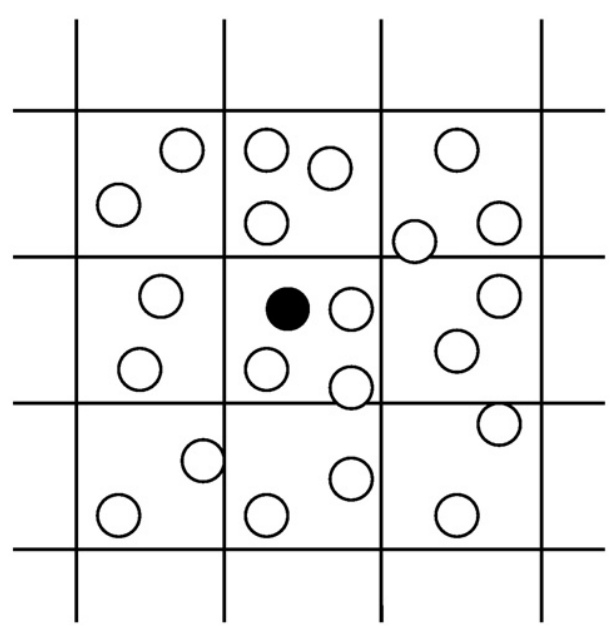

Fig. 1. The structure of neighbor list. (The white particles are stored in the neighbor list of the black particle). 


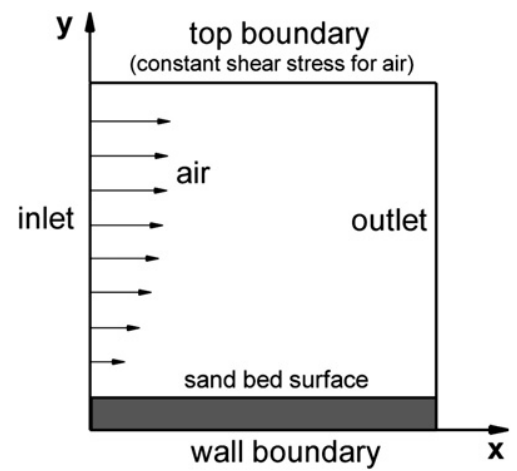

Fig. 2. The structure of computational domain.

air on the top boundary and is independent of the inlet initial velocity of air.

On the wall boundary, the particle-wall collision is modeled in the same manner as particle-particle collision, and the wall is considered as one particle with infinitely large mass, infinitely large diameter and zero velocity vector. For gas, the no-slip condition is used on the wall boundary.

According to the results of Anderson and Haff $(1988,1991)$, the grains due to aerodynamic entrainment can become rare at steady state. Hence, in this paper, the aerodynamic entrainment is neglected, and it is assumed that grain impact is the main mode to generate many new saltation particles at steady state. Grains impacting the sand bed are used to initiate the motion of sand particles on bed surface. Near the bed surface, the motion of particles is determined by inter-particle collisions, no other artificial treatment is applied. In the present simulations, the bottom boundary of the computational domain is the wall boundary and below the sand bed surface. The simulated results are focused on the steady state.

The density of gas is $1.2 \mathrm{~kg} / \mathrm{m}^{3}$, and the dynamic viscosity is $1.785 \times 10^{-5}$ Pas. The particle diameter is $0.33 \mathrm{~mm}$ and particle density is $2650 \mathrm{~kg} / \mathrm{m}^{3}$.

The particle diameter of $0.33 \mathrm{~mm}$ is bigger in order to get larger time step for computational efficiency, so the gas shear stress on the top boundary is chosen as $14.7 \mathrm{~Pa}$ to drive more particles into the air.

The number of particles is 8030 . If the number of particles is insufficient, the statistical number of particles is not enough to achieve a better probability distribution. If the number of particles is more than sufficient, the computational time will be large. Hence, 8030 particles were selected as an optimum. The sand bed at the initial state and steady state for Case 1 is shown in Fig. 3. In the initial state, the sand bed on the bottom boundary includes 8000 particles, and 30 particles above the sand bed are used to impact the bed. From Fig. 3, the height of the initial sand bed is about $5 \mathrm{~mm}$. At the steady state, for Case 1, the sand bed still exists and includes about 7 particle layers, and the height of the sand bed surface is about $2.5 \mathrm{~mm}$.

The friction coefficient is 0.4 , stiffness coefficient is $1500 \mathrm{~N} / \mathrm{m}$, and damping coefficient is 0.002 .

Haff and Anderson (1993) noted that the precise value of friction coefficient has little overall effect on the impact event when the friction coefficient is between 0.25 and 2.0. However, the precise value of friction coefficient is also unclear for natural sand particles. In the present simulations, the friction coefficient is chosen as 0.4.

Haff and Anderson (1993) also reported that the bed response is insensitive to stiffness ranging from 125 to $8000 \mathrm{~N} / \mathrm{m}$. Yuu et al. (1995) studied the particle behavior in a rectangular hopper and found that the value of stiffness had little effect on the particle behavior. Generally, the typical stiffness in the calculations is the order of $1000 \mathrm{~N} / \mathrm{m}$. Another reason is that the particle is softer artificially in order to increase the time step for computational efficiency. In the present simulations, the stiffness coefficient is chosen as $1500 \mathrm{~N} / \mathrm{m}$.

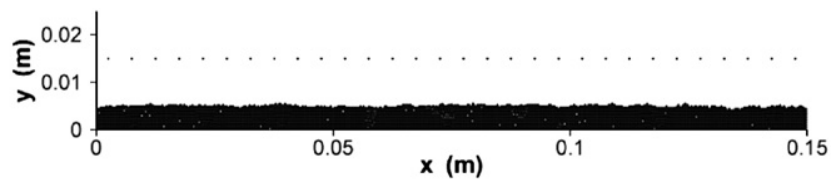

(a) at initial state

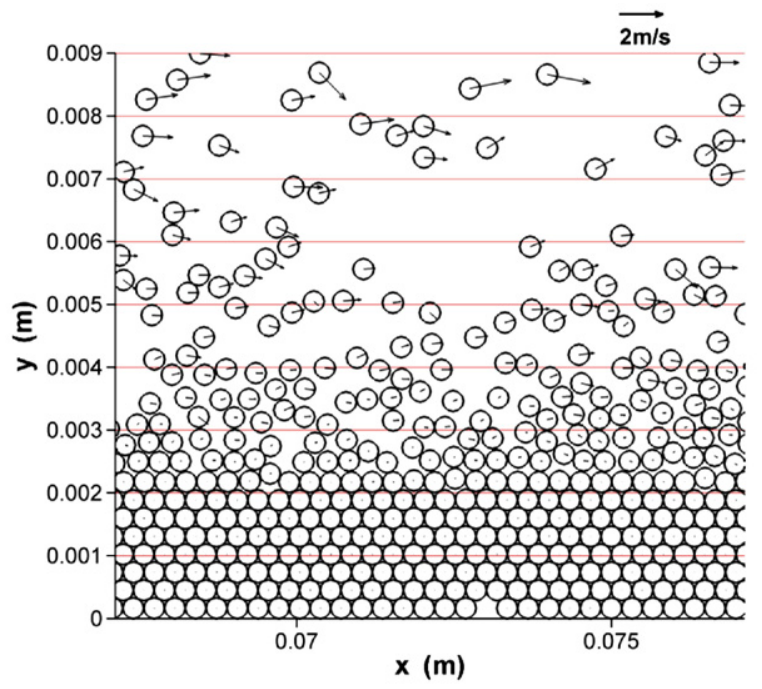

(b) at steady state

Fig. 3. The state of sand bed for Case 1.

The choice of damping coefficient is to obtain the expected restitution coefficient. For the linear spring-damping model, the restitution coefficient is (e.g., Kuo et al., 2002):

$e=\exp \left(\frac{-\eta \pi}{4 m_{p} k_{s}-\eta^{2}}\right)$

where $e$ is the restitution coefficient; $k_{\mathrm{s}}$ and $\eta$ are the stiffness and damping coefficient, respectively; and $m_{p}$ is the mass of particle.

Haff and Anderson (1993) adopted the restitution coefficient of 0.7. In the present simulations, the restitution coefficient is also chosen as 0.7. From Eq. (18), the damping coefficient can be computed as 0.002 .

Tsuji et al. (1993) recommended that the time step for particle should be less than one-tenth of the natural oscillation period of a spring-mass system. For the linear spring system, there is:

$\Delta t_{p}<\frac{\pi}{5} \sqrt{\frac{m_{p}}{k_{s}}}$

where $\Delta t_{p}$ is the time step for particles.

From Eq. (19), the bigger particle diameter and the smaller stiffness can increase the time step for particle and improve the computational efficiency. Under the condition of Eq. (19), the time step for particle is chosen as $2.0 \times 10^{-6} \mathrm{~s}$. The computational time step for the fluid is chosen as $2.0 \times 10^{-5} \mathrm{~s}$. That is to say, there are 10 integration steps for particle trajectory in every time step for fluid motion.

Three cases are simulated as follows:

(1) Case1: The computational domain is a two-dimensional rectangular region $0.35 \mathrm{~m}$ high and $0.15 \mathrm{~m}$ wide. No Magnus force is included. 
(2) Case2: The wall at the bottom is a rough wall, i.e., the particle adjacent to the bottom wall is stationary. The other conditions are the same as that of Case 1 .

(3) Case3: The Magnus force is considered. The height of computational domain is $0.5 \mathrm{~m}$. The other conditions are the same as that of Case 1.

\section{Results and discussions}

We mainly focused on the probability distribution pattern rather than the actual magnitude of particle velocity. In the following, the simulated results are only used to explain the probability distribution pattern.

\subsection{Grid independence test}

For Case 1, two grid sizes are considered: $75 \times 100$ and $100 \times 150$, the numbers of grid cell are 7500 and 15,000, respectively.

Fig. 4 shows the comparison of the two meshes for Case 1. In Fig. 4, $u_{f}$ and $u_{p}$ are the horizontal velocities of the fluid and particles, respectively. It can be seen that the profiles of horizontal velocities of fluid and particles are the same for grid sizes $75 \times 100$ and $100 \times 150$. The variation of sand mass flux with height is also similar for grid sizes $75 \times 100$ and $100 \times 150$. Therefore, the simulated results of grid size $75 \times 100$ can be used to analyze the particle velocity distributions.

In the present simulation, for Case 2 , the grid size is $75 \times 100$. For Case 3 , the grid size is $75 \times 125$ since the height of computational domain is higher.

\subsection{Probability distribution of resultant impact and lift-off velocities of saltating particles}

In the impact-entrainment process above sand bed surface, if the vertical velocity of a particle is upward, the particle will be ascending and is considered as the lift-off particle, otherwise it is the impacting particle. The physical parameters of a saltating trajectory are defined in Fig. 5. In Fig. 5, $u_{\mathrm{I}}$ and $u_{\mathrm{L}}$ are the resultant impact and lift-off velocities of saltating grains, respectively, $\alpha_{\mathrm{I}}$ and $\alpha_{\mathrm{L}}$ are the impact and lift-off angles, respectively.

In the simulated results, the particles within $1 \mathrm{~mm}$ height above sand bed surface are used to calculate the resultant impact and lift-off velocity distributions.

Figs. 6 and 7 show the simulated probability density distribution of resultant impact and lift-off velocities of the saltating grains, respectively. In Figs. 6 and 7, $R^{2}$ is the correlation coefficient. It can be seen that the probability distribution can be expressed by a log-normal function. The following equation is used for this log-normal function:

$P(u)=\frac{1}{\sqrt{2 \pi} A u} \exp \left(\frac{-(\ln u-\ln B)^{2}}{2 A^{2}}\right)$

where $P(u)$ is the probability density, $u$ means the resultant impact velocity or resultant lift-off velocity of the saltating grains. $A$ and $B$ are the regression coefficients.

From Figs. 6 and 7, we also see that the simulated probability density distribution is nearly same for Case 1, Case 2 and Case 3. This means that the bottom boundary condition and the Magnus force have no influence on the simulated probability distribution.

The quantitative comparison between the simulated results and the published experiments is difficult, so a qualitative comparison is shown in Fig. 8. In Fig. 8, the $u_{\mathrm{fo}}$ is the free-stream wind velocity in the wind tunnel experiments of Kang et al. (2008b,c). The nondimensional resultant impact velocity of particles is defined by the ratio of resultant impact velocity to its average. The non-dimensional resultant lift-off velocity of particles is defined by the ratio of resultant
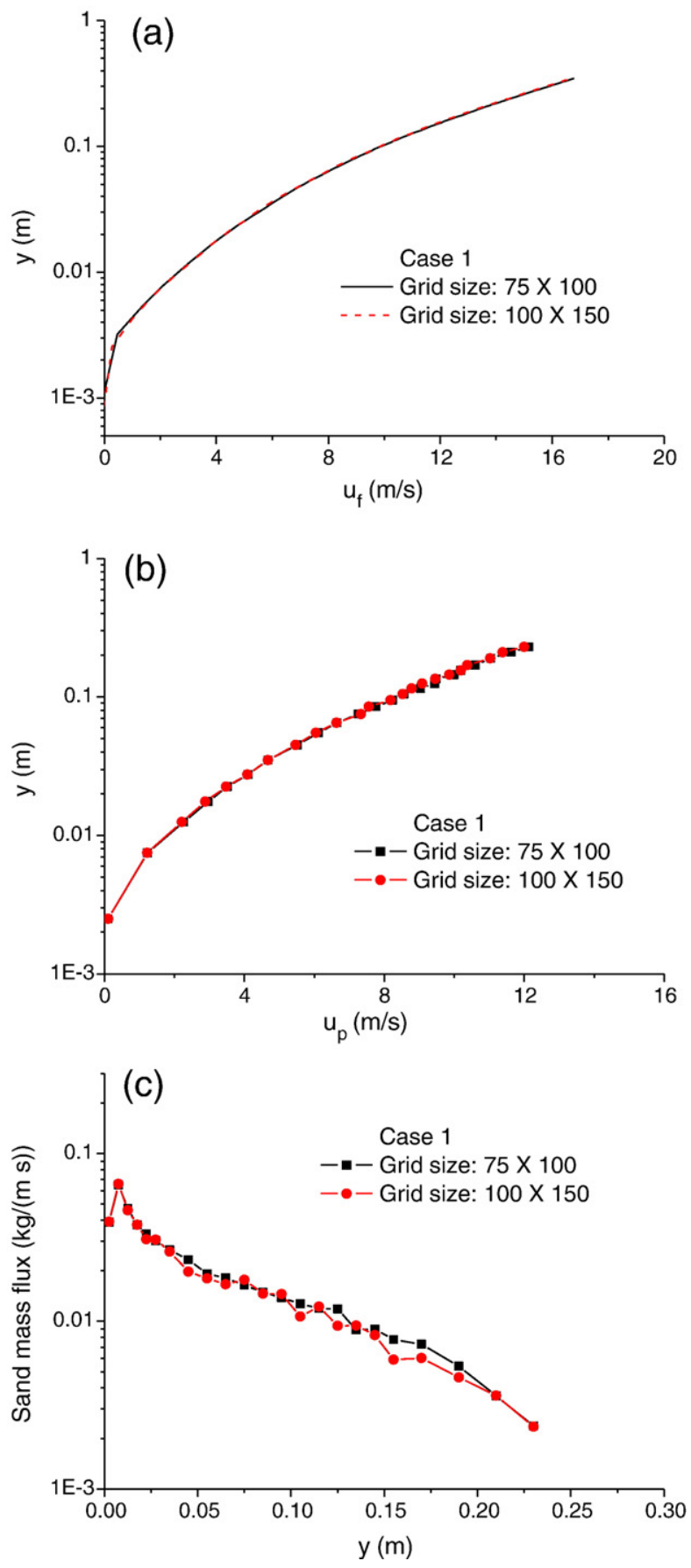

Fig. 4. Result of the comparison of two meshes for Case 1.

lift-off velocity to its average. It can be seen from Fig. 8 that the simulated probability density distributions of the non-dimensional resultant impact and lift-off velocities of particles are accordant with

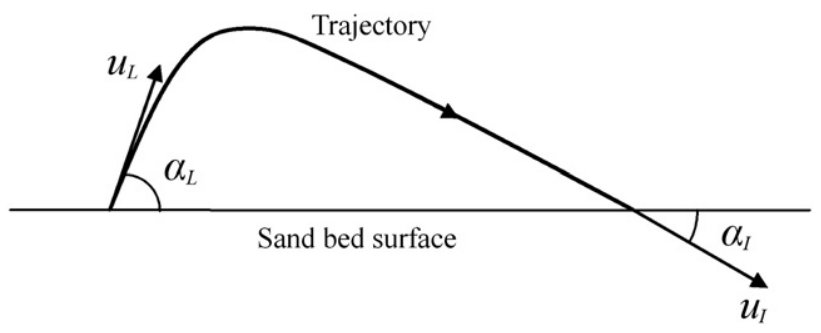

Fig. 5. The physical parameters of a saltating trajectory. 

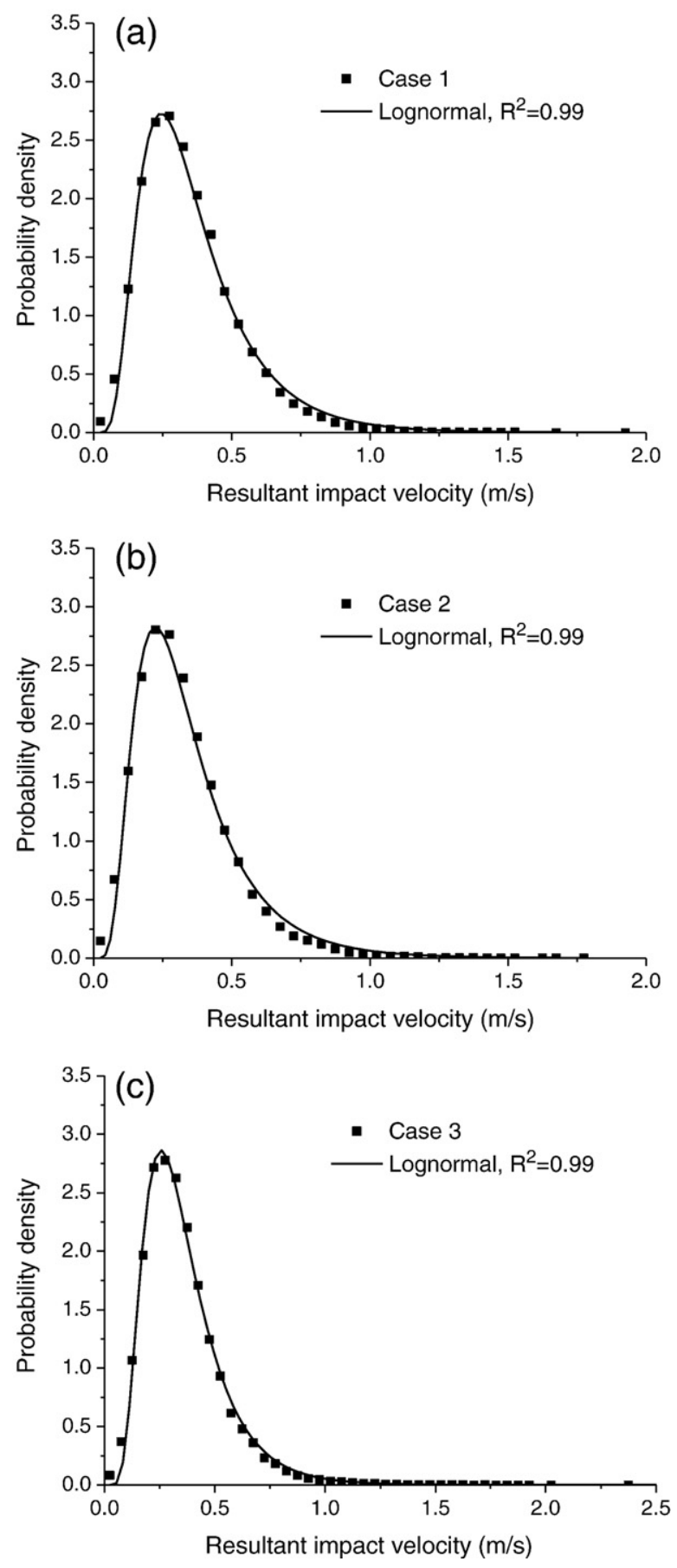

Fig. 6. Simulated distribution of resultant impact velocity.

the experimental results. In the wind tunnel experiments, Kang et al. (2008b) gave the resultant lift-off velocity of the saltating grains as following a log-normal distribution, and Kang et al. (2008c) suggested that the probability distribution of both resultant impact and lift-off velocities can be described by a log-normal function. The present simulated results are qualitatively consistent with these experimental results.

4.3. Probability distribution of impact and lift-off angles of saltating particles

Figs. 9 and 10 denote the simulated probability distribution of impact and lift-off angles of saltating grains, respectively. The probability density distribution of impact angle can be described as
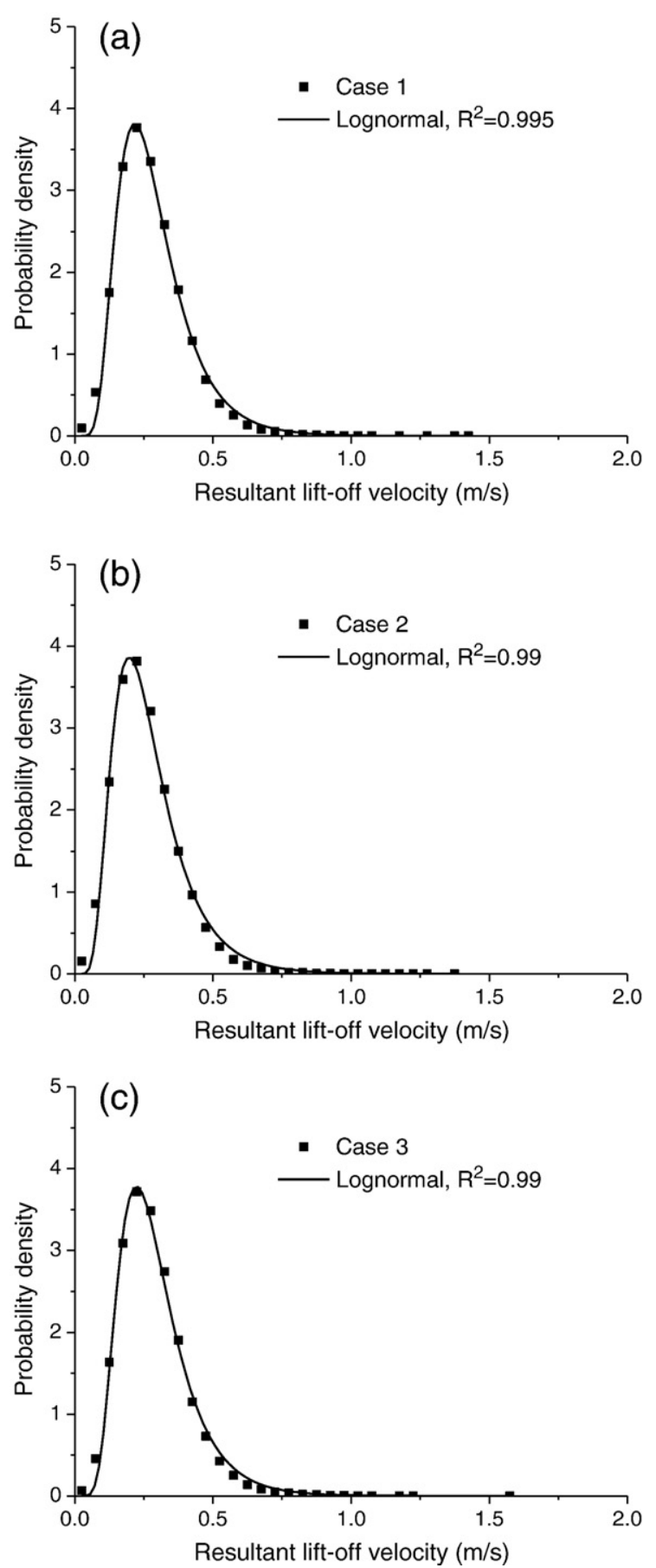

Fig. 7. Simulated distribution of resultant lift-off velocity.

an exponential distribution. The exponential function is expressed as:

$P\left(\alpha_{I}\right)=\frac{1}{A} \exp \left(-\frac{\alpha_{I}}{A}\right)$

where $P\left(\alpha_{\mathrm{I}}\right)$ is the probability density, $\alpha_{\mathrm{I}}$ is the impact angle of the saltating grains. $A$ is the regression coefficient.

The probability density distribution of lift-off angle of the saltating grains seems to deviate from the exponential distribution at the liftoff angle of less than $10^{\circ}$. Near the bed surface, the motion of particles is determined by inter-particle collisions; no other artificial treatment is applied. In the present simulations, the bottom boundary of the computational domain is the wall boundary and below the sand bed 

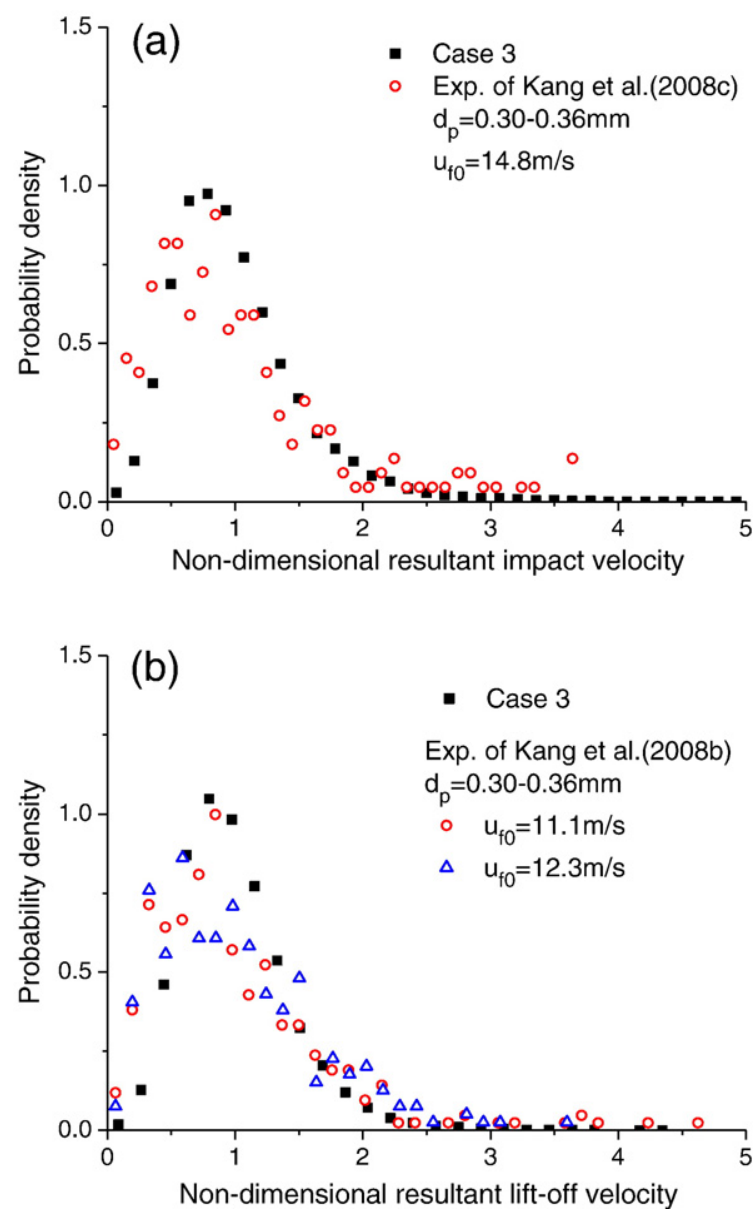

Fig. 8. Qualitative comparison of the simulated and experimental distributions for resultant impact and lift-off velocities.

surface. This deviation may, therefore, be related to this treatment near the bed surface. Further improvement is needed.

In their experiments, Kang et al. (2008b) described the distribution of lift-off angle as an exponential distribution, and Kang et al. (2008c) reported that the probability distribution of both impact and lift-off angles can be described by an exponential function. Fig. 11 shows the qualitative comparison of the simulated and experimental probability density distributions for the non-dimensional impact and lift-off angles. In Fig. 11, the non-dimensional impact angle of saltating particles is defined by the ratio of the impact angle to its average. The nondimensional lift-off angle is defined by the ratio of the lift-off angle to its average. It can be seen from Fig. 11 that the simulated and experimental probability density distribution of the non-dimensional impact angle is consistent and follows an exponential distribution. The simulated probability density distribution of the non-dimensional lift-off angle falls into the region of the experimental data, but deviates from the exponential distribution. Therefore, the present simulation needs to be improved for the distribution of lift-off angle near the sand bed surface.

\subsection{Probability distribution of horizontal and vertical velocities of saltating particles}

In the following, the probability distributions of particle horizontal and vertical velocities are analyzed at different heights. Three selected heights are in the different part of the saltation layer, i.e., $4 \mathrm{~mm}$, $20 \mathrm{~mm}$ and $80 \mathrm{~mm}$.

The particle population is dense near the sand bed surface and sparse at higher heights. The $4 \mathrm{~mm}$ height is selected for the dense particle region near the sand bed surface, and at the lower height, the particle
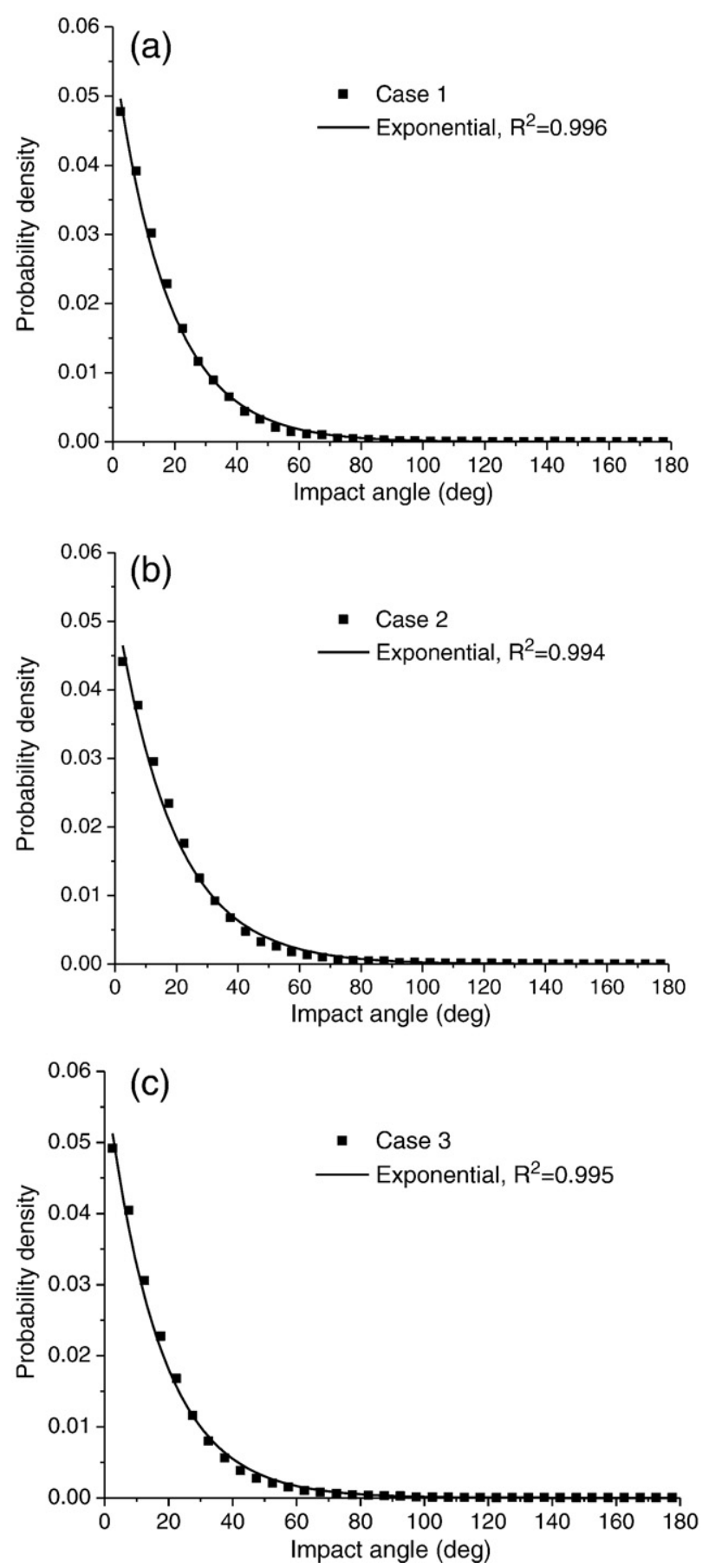

Fig. 9. Simulated distribution of impact angle.

collisions are stronger. In the present simulation, the number of particles above the $80 \mathrm{~mm}$ height is not enough to get a better probability distribution, so the $80 \mathrm{~mm}$ height is selected for the sparse particle region. The $20 \mathrm{~mm}$ height is selected for the middle region between the $4 \mathrm{~mm}$ and $80 \mathrm{~mm}$ heights, and is close to the $4 \mathrm{~mm}$ height in order to get a more statistically significant number of particles.

Fig. 12 is the simulated distribution of particle horizontal velocity at different heights. In Fig. 12, $z$ is defined as the height above the sand bed surface, not the $y$-coordinate of the computational region. It can be seen that the shapes of these histograms have a typical peak.

At a height of $4 \mathrm{~mm}$ in the lower part of the saltation layer, the probability distributions of the particle horizontal velocity are positively skewed. These horizontal velocity distributions are similar to the distributions of grain horizontal velocity at $5 \mathrm{~mm}$ height reported 

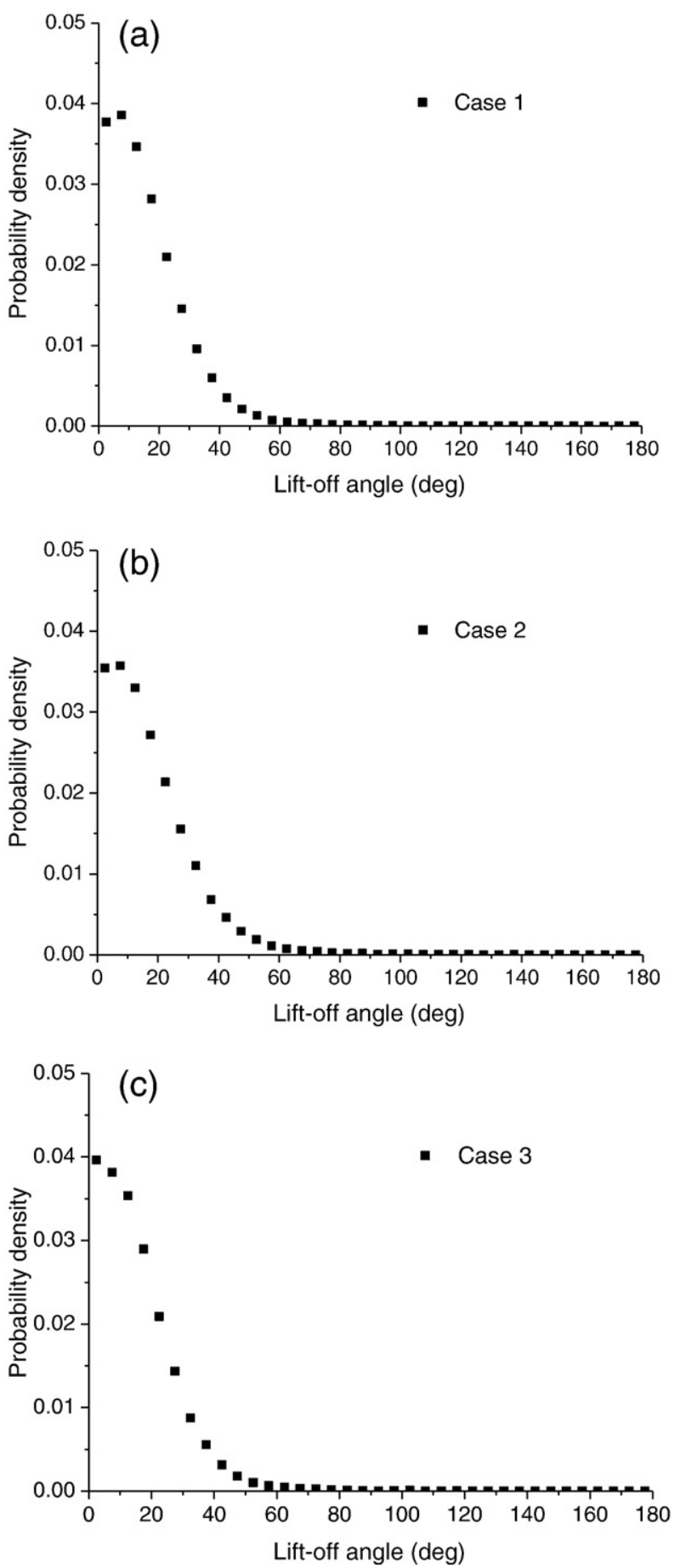

Fig. 10. Simulated distribution of lift-off angle.

by Rasmussen and Sørensen (2005). The reason is explained by Rasmussen and Sørensen (2005) as "during the splash maybe only a single grain may receive a large part of the forward momentum of the impinging grain while several grains will receive some momentum and thus make low, short jumps".

At the middle and upper heights ( $20 \mathrm{~mm}$ and $80 \mathrm{~mm}$ ), the skewness of particle horizontal velocity distributions seems to be relative small.

Fig. 13 denotes the qualitative comparison of the simulated and experimental probability density distributions for non-dimensional particle horizontal velocity. The non-dimensional particle horizontal velocity is defined as the ratio of the particle horizontal velocity to its average. In Fig. 13, $u *$ is the friction velocity in the experiments of Rasmussen and Sørensen (2005).
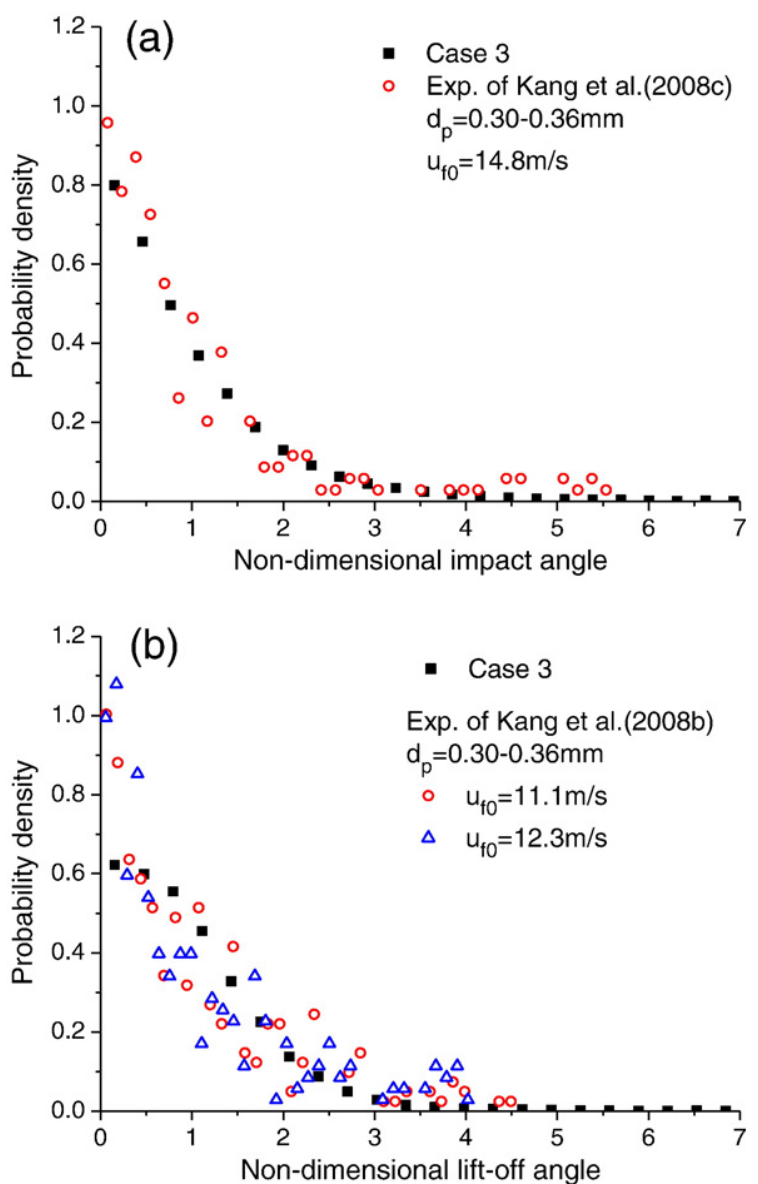

Fig. 11. Qualitative comparison of the simulated and experimental distributions for impact and lift-off angles.

In the experiments of Kang et al. (2008c), the distributions of particle horizontal velocity show a typical single-peak pattern, and at $4 \mathrm{~mm}$ height, the probability distributions of the particle horizontal velocity are asymmetrical and show a positive skewness. It can be seen from Fig. 13 that at the $20 \mathrm{~mm}$ and $80 \mathrm{~mm}$ heights the present simulated probability density distributions of the non-dimensional particle horizontal velocity accord with the experimental results of Kang et al. (2008c), while at the $4 \mathrm{~mm}$ height, only the positive-skew shape of probability density distributions of the non-dimensional particle horizontal velocity is similar between the simulated and experimental results. It is also seen from Fig. 13 that the present simulated probability density distributions of the non-dimensional particle horizontal velocity are qualitatively similar to the experimental results of Rasmussen and Sørensen (2005).

Fig. 14 denotes the simulated distribution of particle vertical velocity at different heights. The particle vertical velocity basically ranges from -2.0 to $2.0 \mathrm{~m} / \mathrm{s}$. The probability distribution of particle vertical velocity has a typical peak.

Fig. 15 shows the qualitative comparison of the simulated and experimental probability density distributions for non-dimensional particle vertical velocity. The non-dimensional particle vertical velocity is defined as the ratio of the particle vertical velocity to the averaged particle horizontal velocity.

In the experiments of Kang et al. (2008c), the probability distribution of particle vertical velocity at different heights can be described as a normal function. While from Fig. 15, the simulated probability density distributions of non-dimensional particle vertical velocity at $4 \mathrm{~mm}$ and $20 \mathrm{~mm}$ heights slightly deviate from the normal function. At $80 \mathrm{~mm}$ height, the simulated distribution of particle vertical velocity can be expressed by a normal distribution function. 

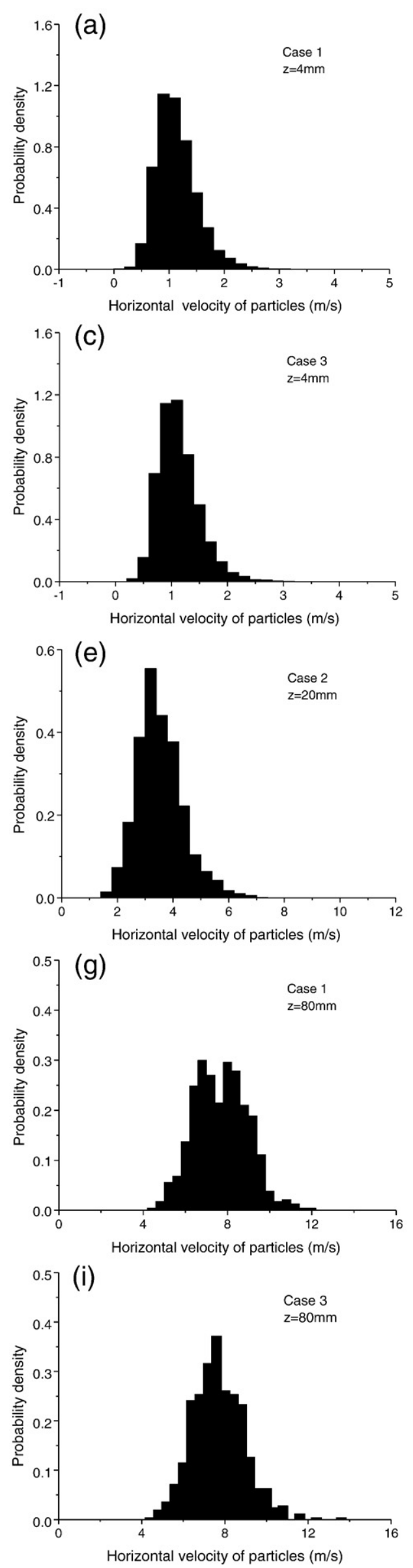
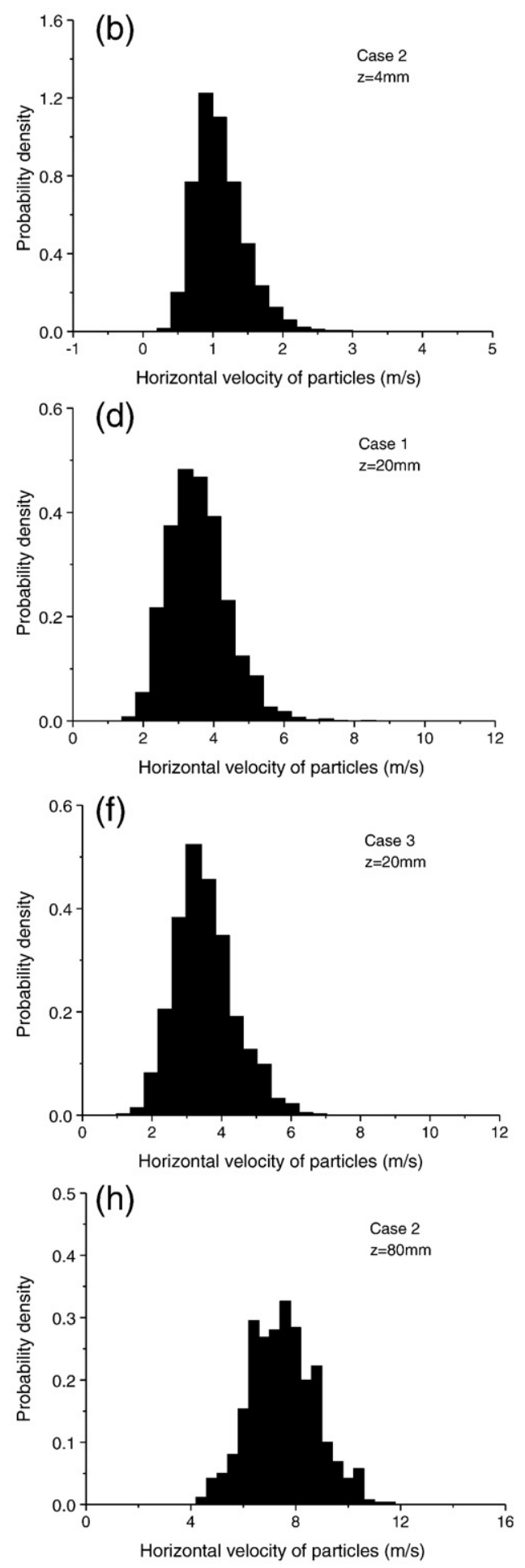

Fig. 12. Simulated distribution of particle horizontal velocity. 

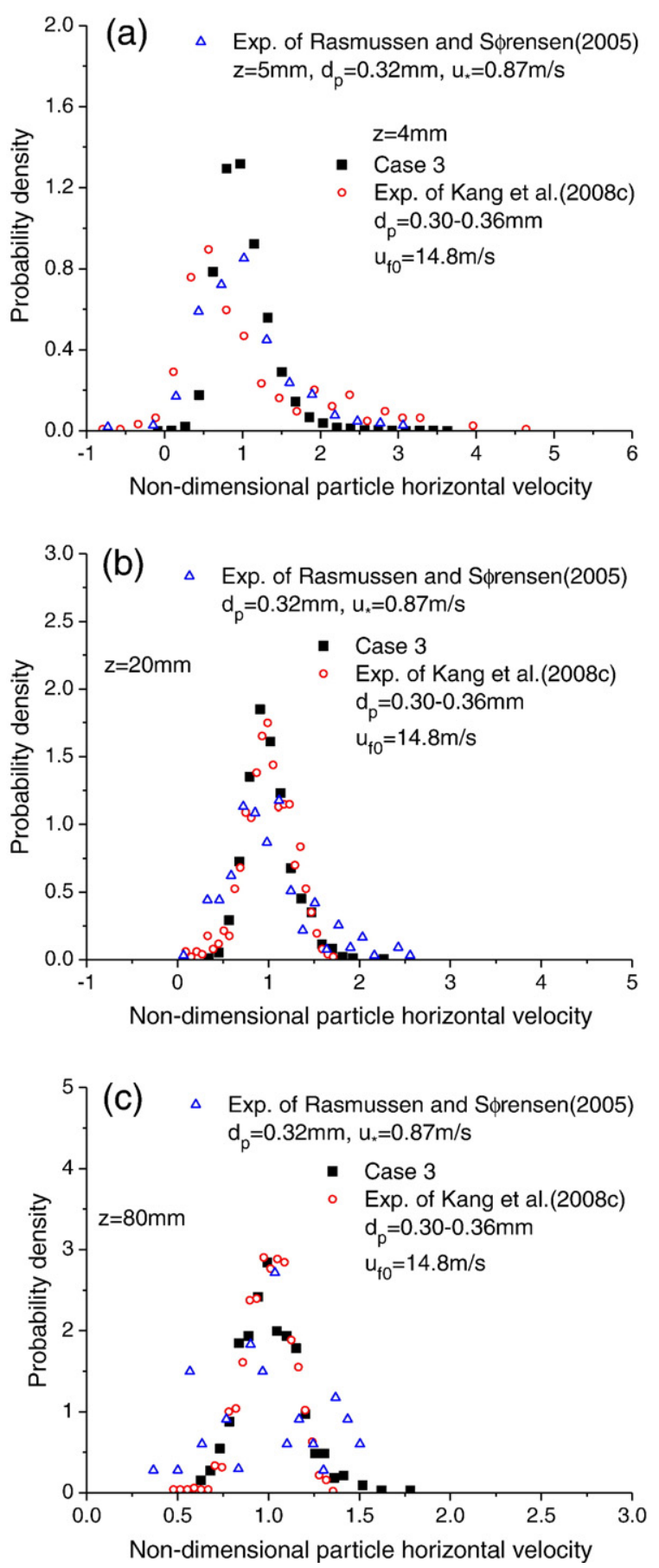

Fig. 13. Qualitative comparison of the simulated and experimental distributions for particle horizontal velocity.

Therefore, the present model should be improved at the lower region of the saltation layer.

It also can be seen from Figs. 6, 7, 9, 10,12 and 14 that the simulated probability density distribution is similar for Case 1, Case 2 and Case 3, hence, the bottom boundary condition and the Magnus force have no obvious effect on the simulated probability density distributions of resultant impact and lift-off velocities, impact and lift-off angles, and the horizontal and vertical velocities of saltating particles. The Magnus force can affect the trajectory of one individual particle, generally increase the saltation height of one clockwise rotational particle and decrease the saltation height of one anti-clockwise rotational particle. In the present simulation, it is found that the number of anti-clockwise rotational particles approximates to that of the clockwise rotational particles.
Therefore, from a statistical view for many particles, the Magnus force has no obvious effect on these probability density distributions.

\section{Further analysis for vertical velocity distribution of ascending particles}

5.1. Vertical velocity distribution of ascending particles on bed surface

It is assumed that:

(1) Each particle is identical with the same mass.

(2) Particle motion is in the equilibrium saltation state.

(3) The vertical component of drag force on the saltating particle is neglected.

(4) The particle collisions in air are neglected.

For the identical particles with identical ejected vertical velocity above the bed surface, as assumed in the saltation model of Owen (1964) and Ungar and Haff (1987), the upward vertical mass flux of particles is conservative in the height direction.

Hence, the upward vertical mass flux of saltation particles at height $z$ can be expressed as:

$q_{v}(z)=m \int_{\sqrt{2 g z}}^{+\infty} n_{0} f\left(v_{0}\right) v_{0} d v_{0}$

where $q_{v}(z)$ is the vertical mass flux of ascending particles at the height $z ; m$ is the mass of a particle; $g$ is gravitational acceleration; $n_{0}$ is the number density of ascending particles on bed surface; $v_{0}$ is the vertical velocity of ascending particles on bed surface, $v_{0}>0$; and $f\left(v_{0}\right)$ is the probability density function of vertical velocity of ascending particles on bed surface.

The above Eq. (22) can be rewritten as:

$q_{v}\left(z\left(v_{0}\right)\right)=q_{v}\left(v_{0}\right)=m \int_{v_{0}}^{+\infty} n_{0} f\left(v_{1}\right) v_{1} d v_{1}$

where $z$ is the function of $v_{0}, z=v_{0}^{2} / 2 g$.

According to Eq. (22), the upward vertical mass flux at bed surface $(z=0)$ is:

$q_{v, 0}=q_{v}(0)=m \int_{0}^{+\infty} n_{0} f\left(v_{0}\right) v_{0} d v_{0}$

In order to calculate the density function $f\left(v_{0}\right)$, the profile of $q_{v}(z)$ is needed. The experimental measurement of $q_{v}(z)$ is very difficult, so the present simulated result is used for analysis. Fig. 16 shows the present simulated profile of vertical mass flux of ascending particles for Case 3. In Fig. 16, the fitted curve is done for $z>0.02 \mathrm{~m}$. The simulated data fit better for an exponential function above $0.02 \mathrm{~m}$ height, but below $0.02 \mathrm{~m}$ height the simulated vertical flux is higher than the vertical flux predicted by the fitted curve.

According to the simulated result (Fig. 16), in this paper, it is assumed that in the whole height region the vertical change of $q_{v}(z)$ decays exponentially with height, as expressed by:

$\frac{q_{v}(z)}{q_{v, 0}}=\exp \left(-a \frac{z}{d_{p}}\right)$

where $a$ is a constant, $a>0, d_{\mathrm{p}}$ is particle diameter.

Insert Eqs. (22) and (23) into Eq. (25), then:

$\frac{m \int_{\sqrt{2 g z}}^{+\infty} n_{0} f\left(v_{0}\right) v_{0} d v_{0}}{q_{v, 0}}=\exp \left(-a z / d_{p}\right)$

$\frac{m \int_{v_{0}}^{+\infty} n_{0} f\left(v_{1}\right) v_{1} d v_{1}}{q_{v, 0}}=\exp \left(-a \frac{v_{0}^{2}}{2 g d_{p}}\right)$ 

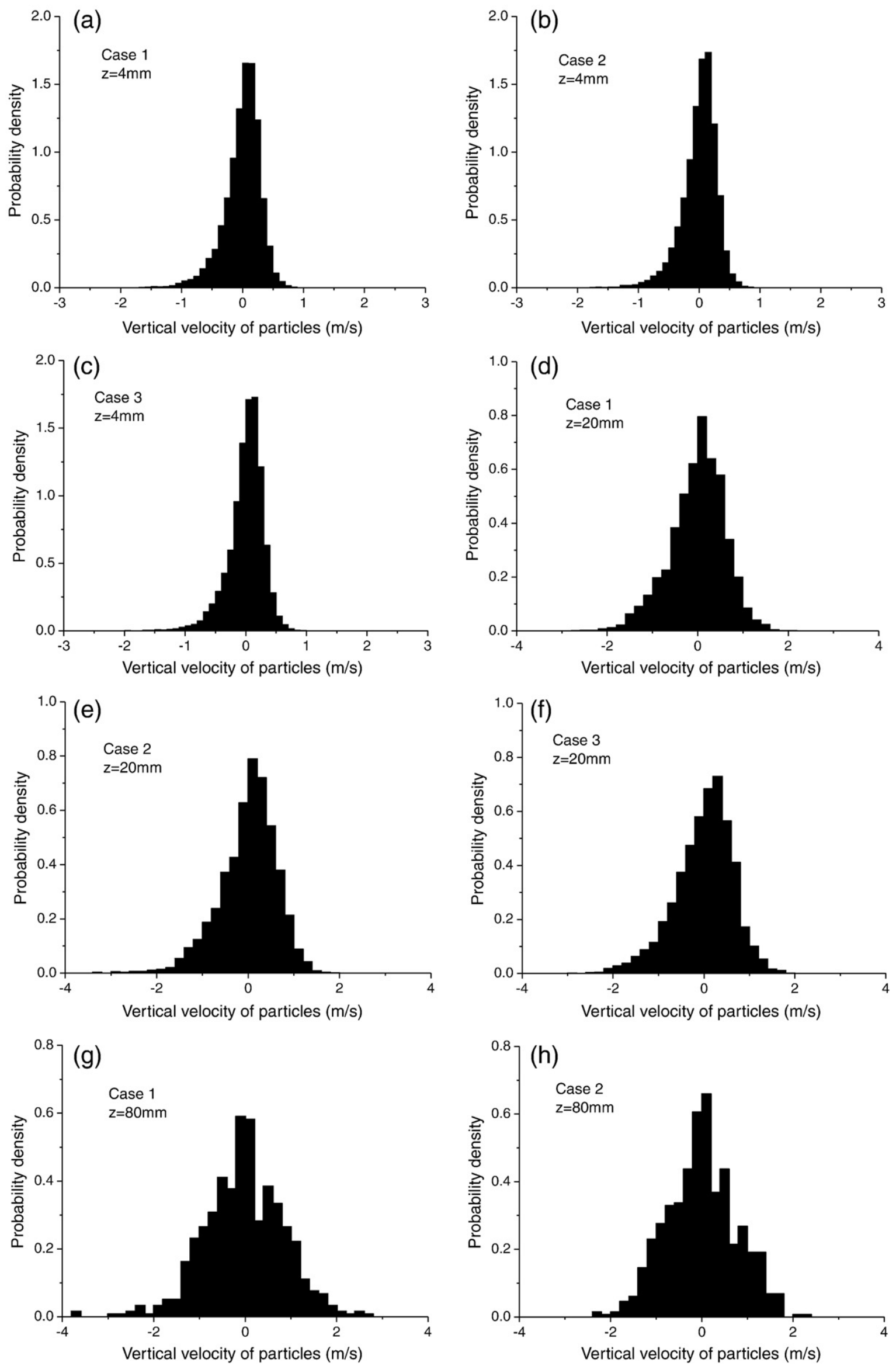

Fig. 14. Simulated distribution of particle vertical velocity. 


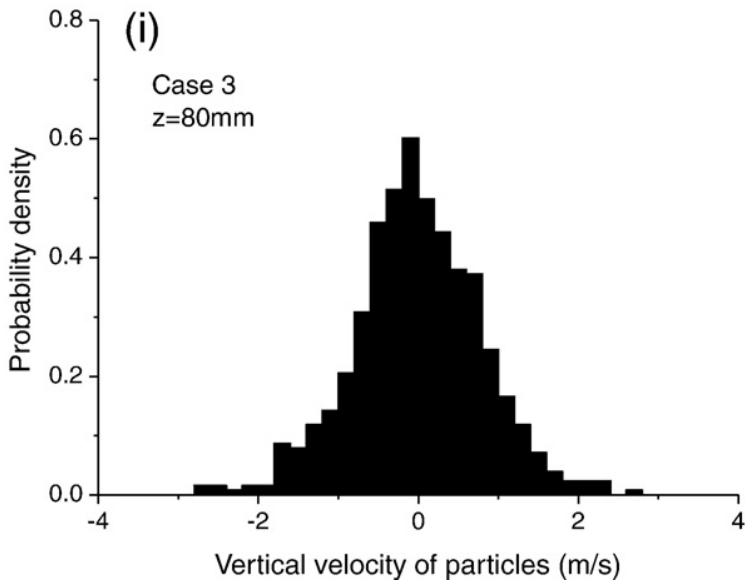

Fig. 14 (continued).

The derivative of Eq. (27) at $v_{0}$ is:

$\frac{-m n_{0} f\left(v_{0}\right) v_{0}}{q_{v, 0}}=-\frac{a v_{0}}{g d_{p}} \exp \left(-a \frac{v_{0}^{2}}{2 g d_{p}}\right)$

Then we get:

$f\left(v_{0}\right)=\frac{a q_{v, 0}}{m n_{0} g d_{p}} \exp \left(-a \frac{v_{0}^{2}}{2 g d_{p}}\right)$

Since $\int_{0}^{+\infty} f\left(v_{0}\right) d v_{0}=1$, then:

$a=\frac{2 g d_{p}}{\pi}\left(\frac{m n_{0}}{q_{v, 0}}\right)^{2}$

The Eq. (29) can be rewritten as:

$f\left(v_{0}\right)=\frac{2 m n_{0}}{\pi q_{v, 0}} \exp \left(-\frac{m^{2} n_{0}^{2}}{\pi q_{v, 0}^{2}} v_{0}^{2}\right)$

Here, we set:

$B=\frac{1}{\pi}\left(\frac{m n_{0}}{q_{v, 0}}\right)^{2}$

Therefore, the probability density function of vertical velocity of ascending particles from the bed surface can be expressed as:

$f\left(v_{0}\right)=\frac{2 \sqrt{B}}{\sqrt{\pi}} \exp \left(-B v_{0}^{2}\right)$

where $B$ is a coefficient, $B>0 . v_{0}>0$.

From Eq. (24), we can get $q_{v, 0}=m n_{0} \overline{v_{0}}$, where $\overline{v_{0}}$ is the mean vertical velocity of ascending particles on bed surface. Hence, the Eq. (33) can be rewritten as:

$f\left(v_{0}\right)=\frac{2}{\pi \overline{v_{0}}} \exp \left(-\frac{v_{0}^{2}}{\pi \bar{v}_{0}^{2}}\right)$

5.2. Vertical velocity distribution of ascending particles at different heights

In the following, the probability density function of vertical velocity of ascending particles at height $z_{1}$ is deduced.
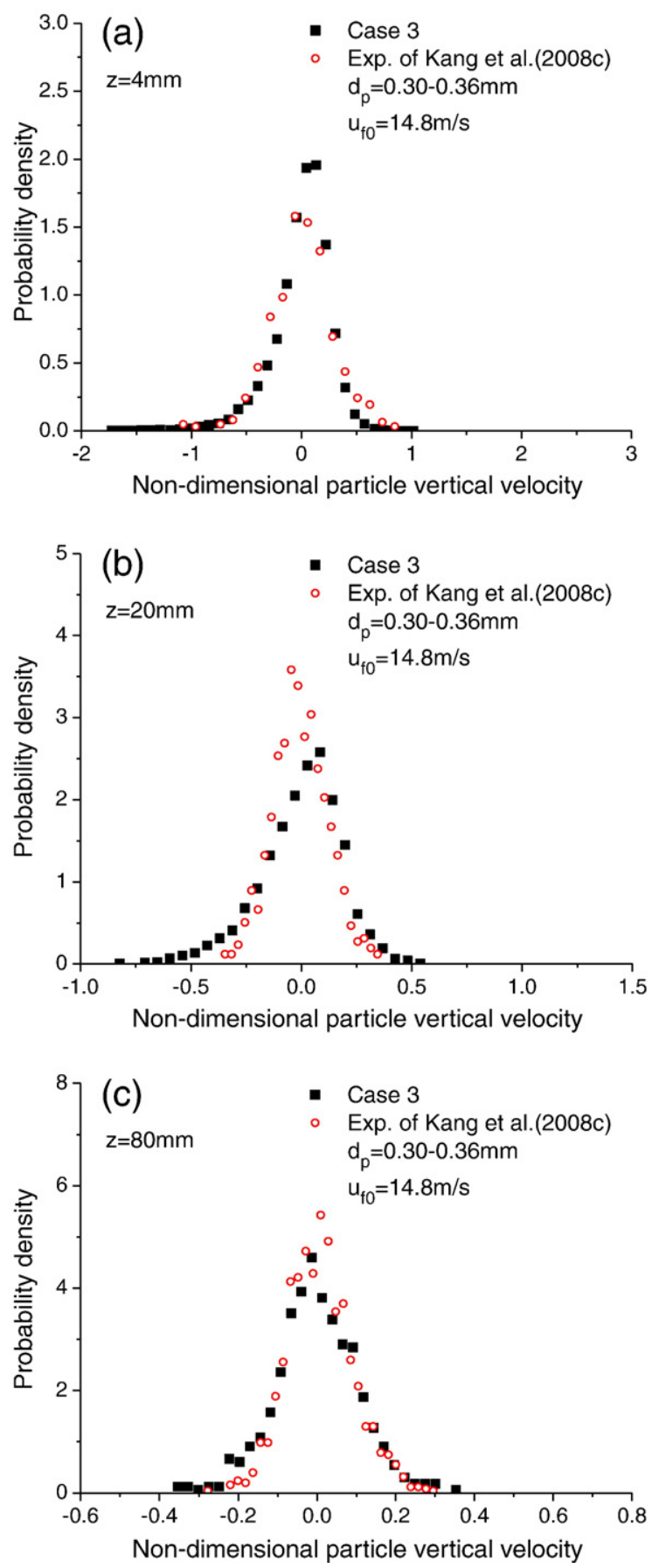

Fig. 15. Qualitative comparison of the simulated and experimental distributions for particle vertical velocity.

The upward vertical mass flux of saltating particles at height $z$ can be expressed as:

$q_{v}(z)=m \int_{\sqrt{2 g\left(z-z_{1}\right)}}^{+\infty} n_{z 1} f\left(v_{z 1}\right) v_{z 1} d v_{z 1}$

where $z>z_{1} . n_{z 1}$ is the number density of ascending particles at height $z_{1} ; v_{z 1}$ is the vertical velocity of ascending particles at height $z_{1}$, $v_{z 1}>0$; and $f\left(v_{z 1}\right)$ is the probability density function of vertical velocity of ascending particles at height $z_{1}$.

The above Eq. (35) can be rewritten as:

$q_{v}\left(z\left(v_{z 1}\right)\right)=q_{v}\left(v_{z 1}\right)=m \int_{v_{z 1}}^{+\infty} n_{z 1} f\left(v_{1}\right) v_{1} d v_{1}$

where $z$ is the function of $v_{z 1}, z=z_{1}+v_{z 1}^{2} / 2 g$. 


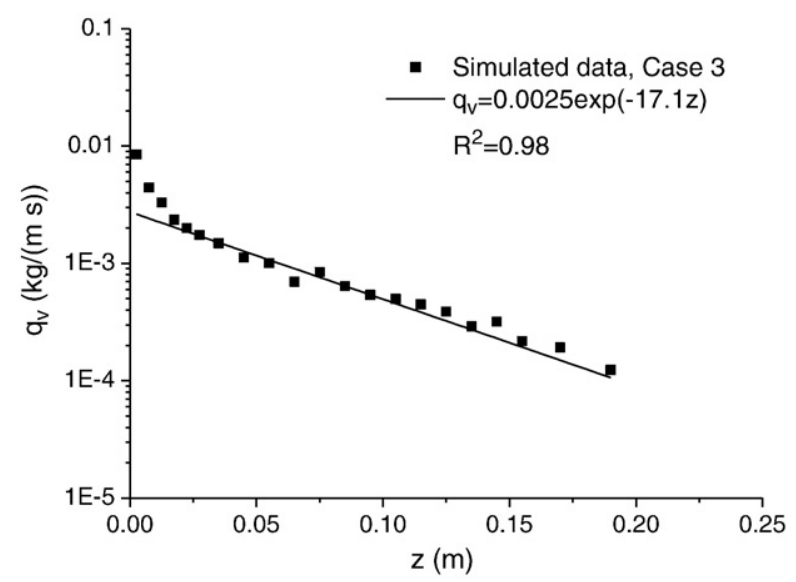

Fig. 16. Simulated profile of vertical mass flux of ascending particles.

From Eq. (25), we get:

$\frac{q_{v}(z)}{q_{v}\left(z_{1}\right)}=\exp \left(-a\left(z-z_{1}\right) / d_{p}\right)$

Insert Eqs. (35) and (36) into Eq. (37), then:

$\frac{m \int_{v_{z 1}}^{+\infty} n_{z 1} f\left(v_{1}\right) v_{1} d v_{1}}{q_{v}\left(z_{1}\right)}=\exp \left(-a \frac{v_{z 1}^{2}}{2 g d_{p}}\right)$
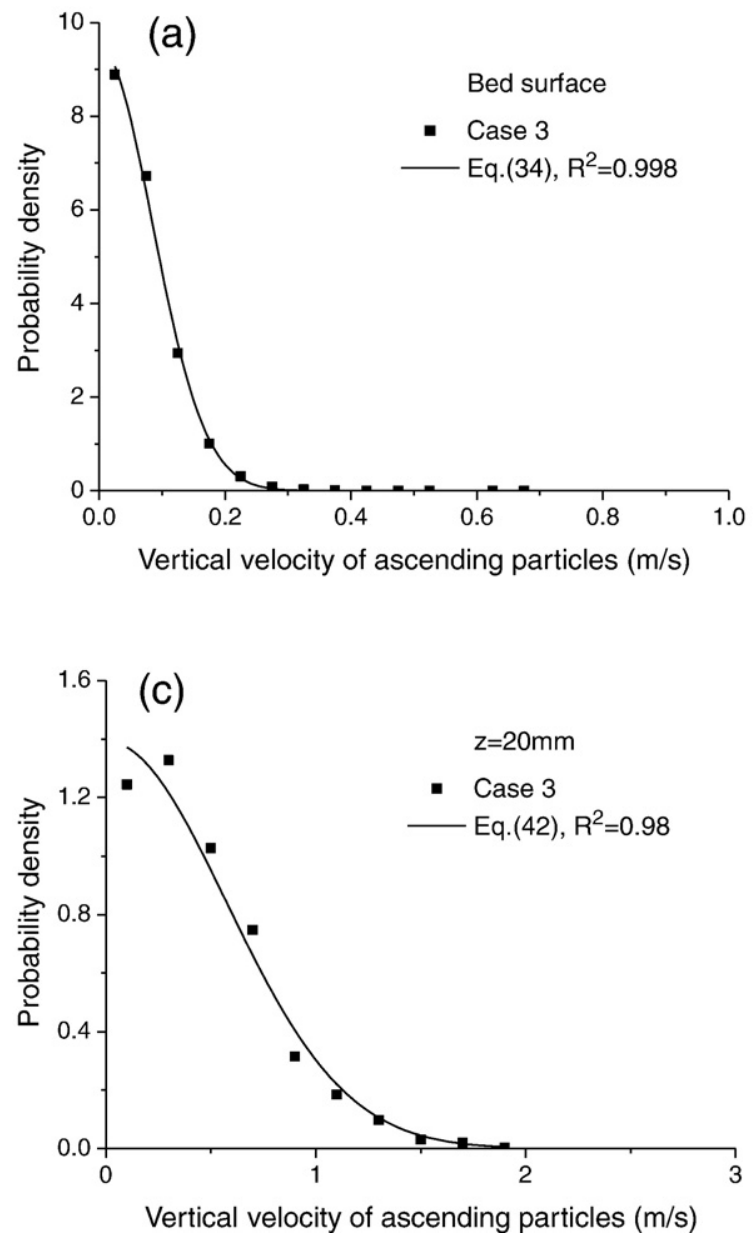

The derivative of Eq. (38) at $v_{z 1}$ is:

$\frac{-m n_{z 1} f\left(v_{z 1}\right) v_{z 1}}{q_{v}\left(z_{1}\right)}=-\frac{a v_{z 1}}{g d_{p}} \exp \left(-a \frac{v_{z 1}^{2}}{2 g d_{p}}\right)$

Then we get:

$f\left(v_{z 1}\right)=\frac{a q_{v}\left(z_{1}\right)}{m n_{z 1} g d_{p}} \exp \left(-a \frac{v_{z 1}^{2}}{2 g d_{p}}\right)$

Therefore, the probability density function of vertical velocity of ascending particles at height $z$ can be expressed as:

$f\left(v_{z}\right)=\frac{2 \sqrt{C}}{\sqrt{\pi}} \exp \left(-C v_{z}^{2}\right)$

where $C$ is a coefficient, $C=\frac{1}{\pi}\left(\frac{m n_{z}}{q_{v}(z)}\right)^{2}>0 . v_{z}>0$.

Since $q_{v}(z)=m n_{z} \bar{v}_{z}$, where $\bar{v}_{z}$ is the mean vertical velocity of ascending particles at height $z$, hence, the Eq. (41) can be rewritten as:

$f\left(v_{z}\right)=\frac{2}{\pi \bar{v}_{z}} \exp \left(-\frac{v_{z}^{2}}{\pi \bar{v}_{z}^{2}}\right)$

It can be seen from Eqs. (34) and (42) that the vertical velocity distribution pattern of ascending particles from the bed surface is the same as that at different heights.
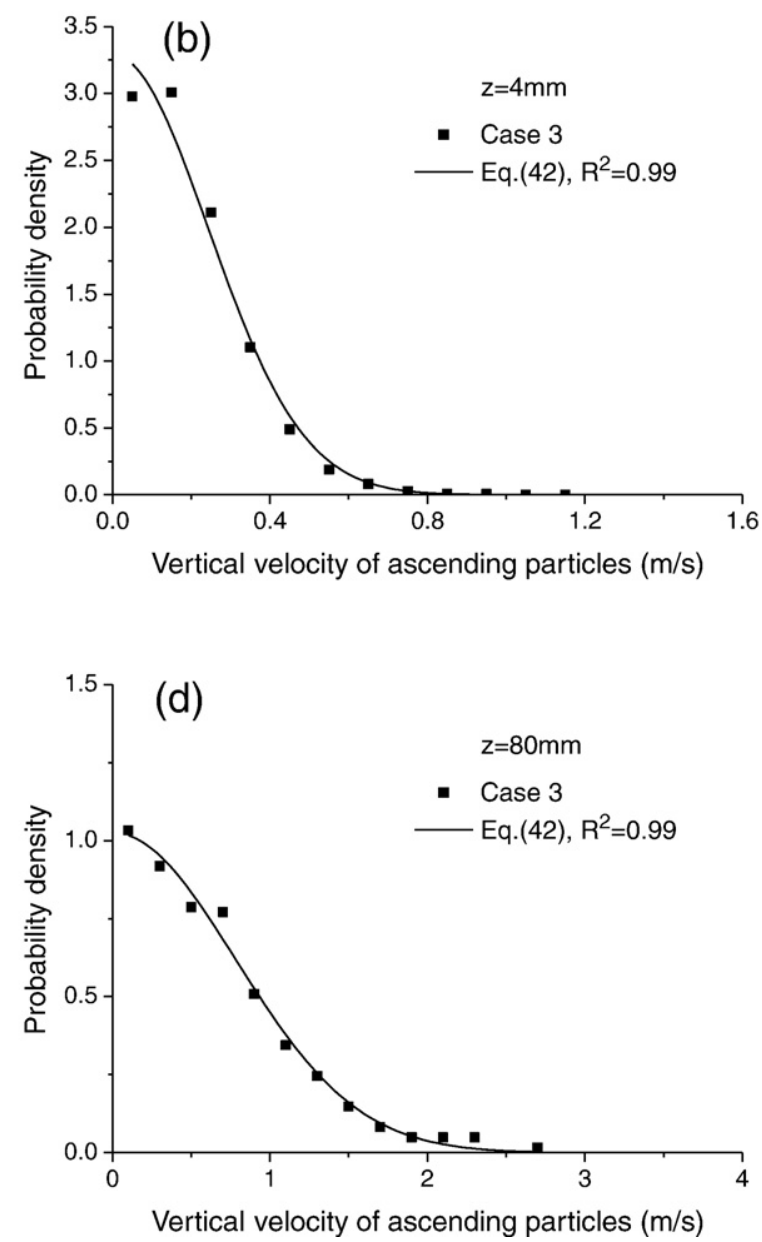

Fig. 17. Simulated vertical velocity distribution of ascending particles. 

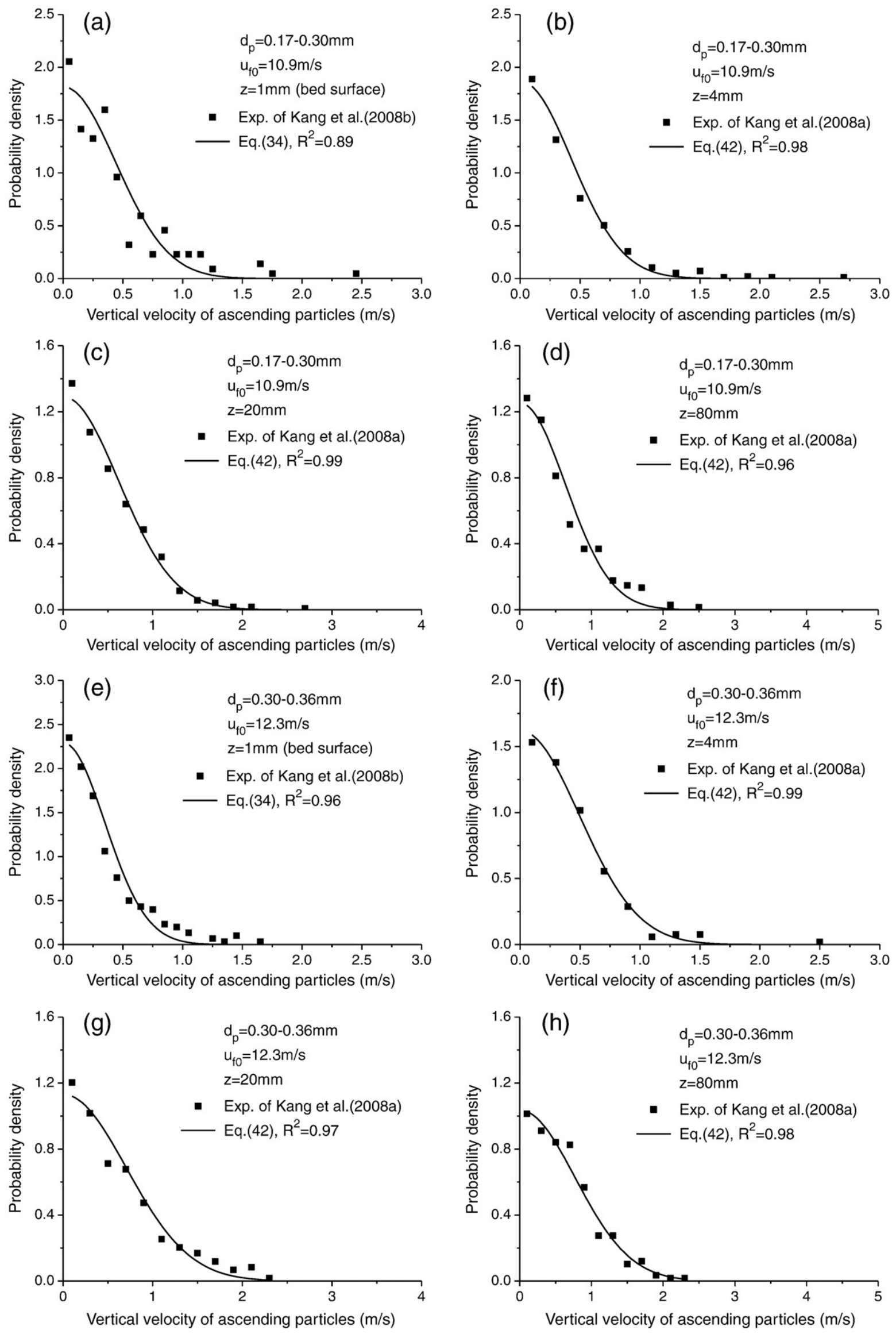

Fig. 18. Experimental vertical velocity distribution of ascending particles. 

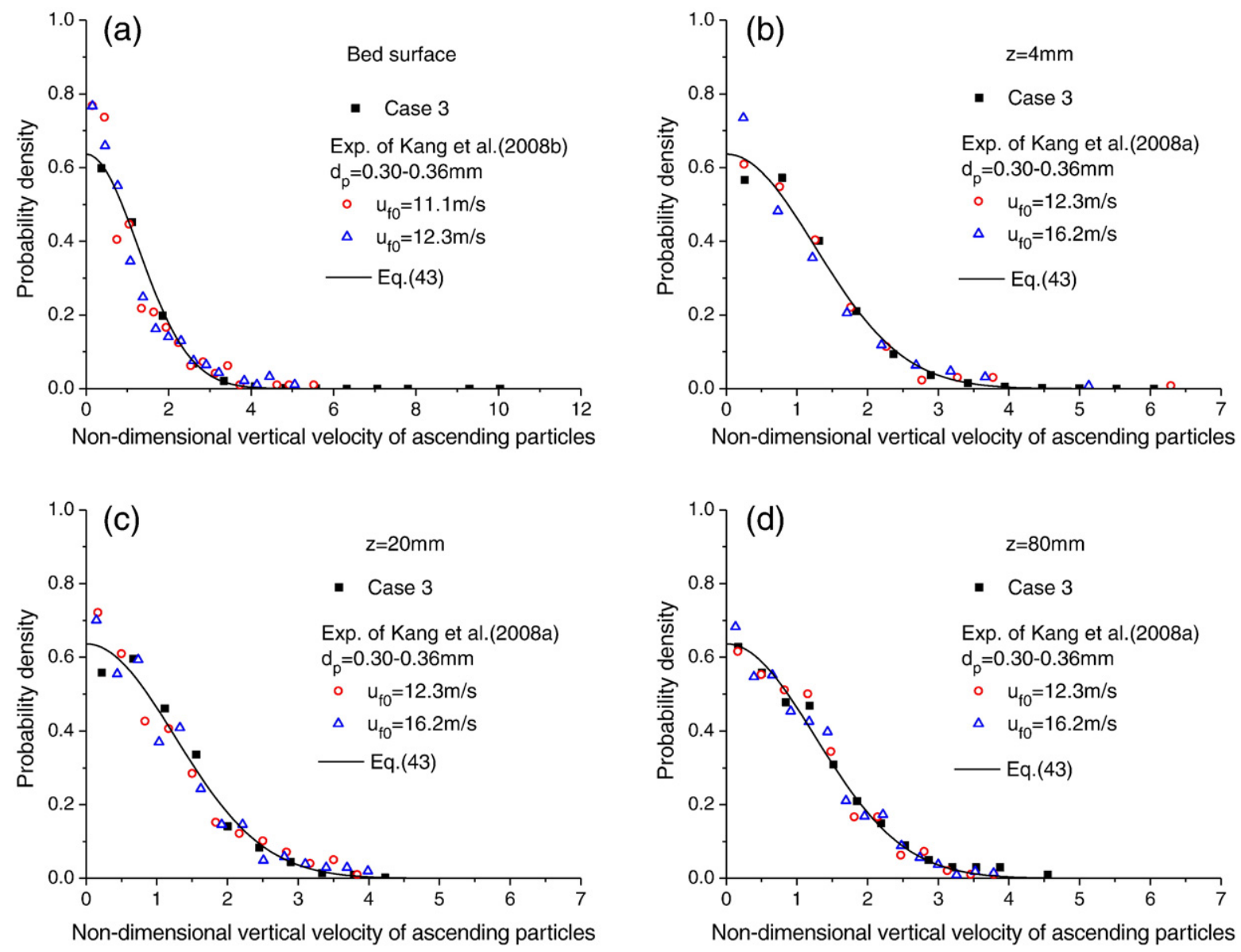

Fig. 19. Qualitative comparison of the simulated and experimental distributions for vertical velocity of ascending particles.

Fig. 17 shows the simulated vertical velocity distribution of acending particles. Fig. 18 is the experimental vertical velocity distribution of ascending particles from Kang et al. (2008a,b). In Fig. 18, the $u_{\mathrm{fo}}$ is the free-stream wind velocity in the wind tunnel experiments of Kang et al. (2008a,b). In the Figs. 17 and 18, the fitting function is Eq. (34) or Eq. (42). It can be seen that the fitted curves predicted by Eqs. (34) and (42) fit better for the simulated and experimental data. The vertical velocity distribution of ascending particles is similar to the right-hand part of a normal distribution function.

It is noted that the probability density function of vertical velocity of ascending particles (i.e., Eqs. (34) and (42)) is not restricted to one fixed particle diameter, hence, in Fig. 18, the experimental results for particle diameter of $0.17-0.30 \mathrm{~mm}$ and $0.30-0.36 \mathrm{~mm}$ are shown.

From the Eqs. (34) and (42), the probability density function of non-dimensional vertical velocity of ascending particles can be expressed as a general form:

$f\left(v_{p \uparrow}^{*}\right)=\frac{2}{\pi} \exp \left(-\frac{v_{p \uparrow}^{*}{ }^{2}}{\pi}\right)$

where $v_{\mathrm{p} \uparrow}^{*}$ is the non-dimensional vertical velocity of ascending particles from a sand bed surface and at different heights, which is defined as the ratio of vertical velocity of ascending particles to its average, i.e., $v_{p \uparrow}^{*}=v_{p \uparrow} / \overline{v_{p} \uparrow}$, where $v_{\mathrm{p} \uparrow}$ and $\overline{v_{p}}$ are vertical velocity of ascending particles and its average, respectively. $f\left(v_{\mathrm{p} \uparrow}^{*}\right)$ is the probability density of non-dimensional vertical velocity of ascending particles from the sand bed surface and at different heights.

Fig. 19 shows the qualitative comparison of the simulated and experimental probability density distributions for the non-dimensional vertical velocity of ascending particles. It can be seen that the simulated probability density distributions for non-dimensional vertical velocity of ascending particles are consistent with the experimental results from Kang et al. (2008a,b). Both the simulated and experimental probability density distributions for non-dimensional vertical velocity of ascending particles comply better with Eq. (43). The probability density function of non-dimensional vertical velocity of ascending particles is independent of diameter of saltating particles, wind strength and height.

\section{Conclusions}

Discrete particle simulation is carried out for particle velocity distribution in saltation. Some features are revealed as follows:

(1) In the impact-entrainment process, the probability density functions of resultant impact and lift-off velocities of saltating particles can be described as a log-normal function, and that of impact angle is an exponential function.

(2) The probability distribution of particle horizontal and vertical velocities at different heights has a typical single peak. In the lower part of saltation layer, the particle horizontal velocity distribution is positively skewed.

(3) The vertical velocity distribution pattern of ascending particles on bed surface and at different heights is identical. The probability density function of vertical velocity of ascending particles is similar to the right-hand part of a normal function. The probability density function of non-dimensional vertical velocity of ascending particles is independent of the diameter of saltating particles, wind strength and height. 
The present discrete particle model can account for the main characteristics of particle velocity distribution in aeolian sand transport. This model is therefore a powerful tool to further study the saltation state in wind-blown sand movement.

\section{Acknowledgements}

This work is supported by National Natural Science Foundation of China (Grant No. 10532030) and the project of State Key Laboratory of Earth Surface Processes and Resource Ecology, Beijing Normal University (Grant No. 070207).

\section{References}

Anderson, R.S., Haff, P.K., 1988. Simulation of eolian saltation. Science 241, 820-823. Anderson, R.S., Haff, P.K., 1991. Wind modification and bed response during saltation of sand in air. Acta Mechanica (suppl. 1), 21-51.

Anderson, R.S., Hallet, B., 1986. Sediment transport by wind: toward a general model Geological Society of America Bulletin 97, 523-535.

Bagnold, R.A., 1941. The Physics of Blown Sand and Desert Dunes. Methuen, London.

Cheng, H., Zou, X.Y., Zhang, C.L., 2006. Probability distribution functions for the initia liftoff velocities of saltating sand grains in air. Journal of Geophysical ResearchAtmospheres 111, D22205.

Crowe, C.T., Sommerfeld, M., Tsuji, Y., 1998. Multiphase Flows with Droplets and Particles. CRC Press, Boca Raton.

Di Felice, R., 1994. The voidage function for fluid-particle interaction systems. International Journal of Multiphase Flow 20, 153-159.

Dong, Z.B., Liu, X.P., Li, F., Wang, H.T., Zhao, A.G., 2002. Impact-entrainment relationship in a saltating cloud. Earth Surface Processes and Landforms 27, 641-658.

Dong, Z.B., Liu, X.P., Wang, X.M., Li, F., Zhao, A.G., 2004. Experimental investigation of the velocity of a sand cloud blowing over a sandy surface. Earth Surface Processes and Landforms 29, 343-358.

Greeley, R., Blumberg, D.G., Williams, S.H., 1996. Field measurements of the flux and speed of wind-blown sand. Sedimentology 43, 41-52.

Haff, P.K., Anderson, R.S., 1993. Grain scale simulations of loose sedimentary beds: the example of grain-bed impacts in aeolian saltation. Sedimentology 40, 175-198.

Happel, J., Brenner, H., 1973. Low Reynolds Number Hydrodynamics. Noordhoff International Publishing, Leyden.

Huang, N., Zheng, X.J., Zhou, Y.H., Van Pelt, R.S., 2006. Simulation of wind-blown sand movement and probability density function of liftoff velocities of sand particles. Journal of Geophysical Research-Atmospheres 111, D20201.

Kang, L.Q., Guo, L.J., 2006. Eulerian-Lagrangian simulation of aeolian sand transport Powder Technology 162, 111-120.

Kang, L.Q., Guo, L.J., Gu, Z.M., Liu, D.Y., 2008a. Wind tunnel experimental investigation of sand velocity in aeolian sand transport. Geomorphology 97, 438-450.
Kang, L.Q., Guo, L.J., Liu, D.Y., 2008b. Reconstructing the vertical distribution of the aeolian saltation mass flux based on the probability distribution of lift-off velocity. Geomorphology 96, 1-15.

Kang, L.Q., Guo, L.J., Liu, D.Y., 2008c. Experimental investigation of particle velocity distributions in windblown sand movement. Science in China Series G: Physics Mechanics and Astronomy 51, 986-1000.

Kuo, H.P., Knight, P.C., Parker, D.J., Tsuji, Y., Adams, M.J., Seville, J.P.K., 2002. The influence of DEM simulation parameters on the particle behaviour in a V-mixer. Chemical Engineering Science 57, 3621-3638.

McEwan, I.K., Willetts, B.B., 1991. Numerical model of the saltation cloud. Acta Mechanica (suppl.1), 53-66.

McEwan, I.K., Willetts, B.B., 1993. Adaptation of the near-surface wind to the development of sand transport. Journal of Fluid Mechanics 252, 99-115.

Mikami, T., Kamiya, H., Horio, M., 1998. Numerical simulation of cohesive powder behavior in a fluidized bed. Chemical Engineering Science 53, 1927-1940.

Nalpanis, P., Hunt, J.C.R., Barrett, C.F., 1993. Saltating particles over flat beds. Journal of Fluid Mechanics 251, 661-685.

Namikas, S.L., 2003. Field measurement and numerical modelling of aeolian mass flux distributions on a sandy beach. Sedimentology 50, 303-326.

Owen, P.R., 1964. Saltation of uniform grains in air. Journal of Fluid Mechanics 20, 225-242.

Rasmussen, K.R., Sørensen, M., 2005. Dynamics of particles in aeolian saltation. Powders and Grains, vol. 2. Balkema A.A., Rotterdam, pp. 967-971.

Raupach, M.R., 1991. Saltation layers, vegetation canopies and roughness lengths. Acta Mechanica (Suppl. 1), 83-96.

Rubinow, S.I., Keller, J.B., 1961. The transverse force on a spinning sphere moving in a viscous fluid. Journal of Fluid Mechanics 11, 447-459.

Sørensen, M., 1991. An analytic model of wind-blown sand transport. Acta Mechanica (Suppl. 1), 67-81.

Spies, P.-J., McEwan, I.K., 2000. Equilibration of saltation. Earth Surface Processes and Landforms 25, 437-453.

Spies, P.-J., McEwan, I.K., Butterfield, G.R., 2000. One-dimentional transitional behaviour in saltation. Earth Surface Processes and Landforms 25, 505-518.

Tsuji, Y., Kawaguchi, T., Tanaka, T., 1993. Discrete particle simulation of twodimensional fluidized bed. Powder Technology 77, 79-87.

Ungar, J.E., Haff, P.K., 1987. Steady state saltation in air. Sedimentology 34, 289-299.

Willetts, B.B., Rice, M.A., 1986. Collisions in aeolian saltation. Acta Mechanica 63, 255-265.

Xu, B.H., Yu, A.B., 1997. Numerical simulation of the gas-solid flow in a fluidized bed by combining discrete particle method with computational fluid dynamics. Chemical Engineering Science 52, 2785-2809.

Yuu, S., Abe, T., Saitoh, T., Umekage, T., 1995. Three-dimensional numerical simulation of the motion of particles discharging from a rectangular hopper using distinct element method and comparison with experimental data (effects of time steps and material properties). Advanced Powder Technology 6, 259-269.

Zhang, W., Kang, J.H., Lee, S.J., 2007. Tracking of saltating sand trajectories over a flat surface embedded in an atmospheric boundary layer. Geomorphology 86, 320-331.

Zou, X.Y., Wang, Z.L., Hao, O.Z., Zhang, C.L., Liu, Y.Z., Dong, G.R., 2001. The distribution of velocity and energy of saltating sand grains in a wind tunnel. Geomorphology 36, 155-165. 